\title{
Activités
}

18-2 | 2021

Espace et temps de l'activité / Travail, Territoire et politique

\section{Identifier des routines interactionnelles dans le travail des éducateurs de l'enfance : le cas des arrivées des enfants dans les structures d'accueil}

Identifying interactional routines in the work of early childhood educators: the case of children's drop-offs at childcare facilities

Marianne Zogmal et Laurent Filliettaz

\section{(2) OpenEdition}

Journals

Édition électronique

URL : https://journals.openedition.org/activites/6885

DOI : 10.4000/activites.6885

ISSN : 1765-2723

Éditeur

ARPACT - Association Recherches et Pratiques sur les ACTivités

Référence électronique

Marianne Zogmal et Laurent Filliettaz, « Identifier des routines interactionnelles dans le travail des éducateurs de l'enfance : le cas des arrivées des enfants dans les structures d'accueil », Activités [En ligne], 18-2 | 2021, mis en ligne le 15 octobre 2021, consulté le 07 avril 2022. URL : http:// journals.openedition.org/activites/6885; DOI : https://doi.org/10.4000/activites.6885

Ce document a été généré automatiquement le 7 avril 2022.

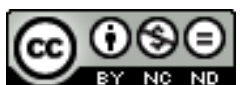

Activités est mis à disposition selon les termes de la licence Creative Commons Attribution - Pas d'Utilisation Commerciale - Pas de Modification 4.0 International. 


\section{Identifier des routines} interactionnelles dans le travail des éducateurs de l'enfance : le cas des arrivées des enfants dans les structures d'accueil

Identifying interactional routines in the work of early childhood educators: the case of children's drop-offs at childcare facilities

Marianne Zogmal et Laurent Filliettaz

\section{NOTE DE L'ÉDITEUR}

Article soumis le 05/02/2021, accepté le 19/04/2021

\section{Introduction}

1 Le travail au quotidien des éducatrices et éducateurs de l'enfance a longtemps constitué une réalité méconnue et occultée par d'innombrables représentations sociales et attentes institutionnelles. Peut-être parce qu'il semble associé à des dispositions naturelles ou acquises spontanément - celles de savoir éduquer des enfants - il n'a pendant longtemps pas donné lieu à un effort de recherche équivalant à celui déployé à propos d'autres métiers de l'éducation (Lussi Borer, Durand, \& Yvon, 2015). Pourtant, on assiste depuis quelques années à un intérêt renouvelé pour l'analyse du «travail» des professionnels de la petite enfance et pour la compréhension du «métier » et des processus de professionnalisation qui y sont à l'œuvre (Pirard, 2007). Dans les pays francophones, des travaux de recherche émergent progressivement pour montrer 
comment les pratiques professionnelles dans le champ de l'accueil extrafamilial s'accomplissent, se transmettent et s'apprennent.

Des observations à caractère ethnographique soulignent par exemple les tensions vécues par les professionnels de l'éducation de l'enfance entre les normes éthiques que poursuivent les acteurs et les conditions à la fois pratiques et organisationnelles dans lesquelles s'accomplit leur activité au quotidien (Ulmann, 2013) ou leur formation professionnelle (Ulmann, 2017). Les logiques d'interdépendance qui sous-tendent le travail éducatif se dégagent également comme une caractéristique déterminante du métier. On le sait bien, le travail éducatif « ne se fait pas tout seul ». D'abord parce qu'il mobilise chez les professionnels une mise en œuvre de ressources dans des situations nécessairement singulières et souvent marquées par l'incertitude. Et ensuite parce qu'il repose sur une distribution de ces ressources et un accomplissement nécessairement collectif. Il s'agit d'abord de distribuer son attention auprès d'une pluralité d'enfants pris en charge simultanément. Il s'agit ensuite de coordonner son travail avec celui d'autres professionnels, au sein d'un collectif et d'un « système compétent » (Pirard \& Barbier, 2012). Et il s'agit enfin de construire des rapports de partenariat avec d'autres acteurs, comme les parents notamment, dans une logique de "co-éducation » et de symétrie des rapports sociaux (Bonabesse \& Blanc, 2013 ; Bouve, 2009 ; Brougère, 2010, 2015). Ces échanges donnent lieu à un intense travail interactionnel. Ils sont fortement médiatisés par l'usage du langage et le recours à des pratiques de communication. Ainsi, la compétence dans le champ de l'éducation de l'enfance s'exprime largement dans et à travers les interactions. Elle requiert ainsi la mobilisation et le développement d'une " compétence d'interaction", définie comme la capacité des acteurs de pouvoir configurer collectivement les ressources permettant de s'engager dans des pratiques sociales de manière reconnue comme légitime (Pekarek, Bangerter, de Weck, \& Fillettaz, 2017; Young \& Miller, 2004). Cette compétence d'interaction doit non seulement être mise en œuvre dans le quotidien du travail et acquise dans les expériences de formation professionnelle initiale (Alonso Vilches \& Pirard, 2016; Filliettaz \& Zogmal, 2020; Zogmal 2020a, 2020b). Mais elle peut également être entretenue et développée au cours de l'expérience, par des dispositifs de formation continue fondés sur une analyse interactionnelle du travail (Fillietaz, 2014; Garcia \& Filliettaz, 2020 ; Zogmal \& Durand, 2020).

3 Le travail au quotidien des professionnels de l'éducation a souvent été décrit dans son caractère « discrétionnaire » (Pastré, 2011 ; Vinatier, 2013) et en référence à la rareté des ressources permettant aux acteurs de comprendre comment ils peuvent s'aligner aux prescriptions et à ce qui est attendu d'eux. À de nombreux égards, travailler au sein d'une institution de la petite enfance consiste à faire face à des situations imprévues et à s'accommoder aux circonstances toujours singulières qui se présentent quotidiennement. Pourtant, force est de constater que dans cet environnement hautement dynamique, collectif et distribué, des routines existent, qui agissent comme des repères stables, aussi bien pour les professionnels que pour les usagers des institutions concernées. Les rythmes de vie d'une institution de la petite enfance sont fortement organisés. Ils sont ponctués d'activités ou de « situations » reconnues comme typiques, dont la catégorisation transparait clairement dans les discours institutionnels ou les plans d'études des programmes de formation (PEC, 2015). On dit généralement des activités éducatives qu'elles peuvent être "structurées" ou "libres ", qu'elles alternent avec des moments de « réunions », qu'elles sont ponctuées de «repas » et de 
temps de "soins ». Les routines qui s'établissent dans la mise en place de ces activités et les conditions dans lesquelles elles s'articulent et alternent répondent à des besoins de repères pour les enfants dans le cadre de leur développement. Mais ces routines agissent également comme des éléments structurant sur le plan organisationnel. Ils servent de ressources dans le cadre de l'organisation du travail et sont utilisés comme un instrument de coordination et de distribution des tâches au sein des équipes éducatives.

4 Parmi les routines et les situations d'interaction qui ponctuent le quotidien des institutions éducatives, les temps d'accueils et de départs tiennent une place particulièrement proéminente. En effet, toute prise en charge d'un enfant dans contexte institutionnel est initiée par son arrivée et clôturée par son départ. Ces temps se répètent quotidiennement. Ils agissent comme des espaces de transition entre la sphère familiale privée et la sphère publique de l'institution éducative. Les enfants sont généralement accompagnés de leurs parents ou plus rarement d'autres membres de leur famille au moment des arrivées et des départs. À ce titre, ces situations de transition servent aussi d'espace de rencontre entre les professionnels et les familles. On y transmet des informations, on y partage des observations, on y échange des conseils. Or on sait que cette composante de l'activité, orientée vers la construction d'une relation de partenariat avec les parents, ne constitue pas un ingrédient marginal du travail, mais tend aujourd'hui à être considérée comme une part intégrante et légitime de la professionnalité attendue des éducateurs (Rayna, Rubio, \& Scheu, 2000). Les arrivées et les départs constituent aussi des moments éphémères, fugaces et accomplis sous contrainte de temps. On s'y réfère parfois comme des interactions accomplies "sur le pas de la porte " (Chatelain-Gobron, 2014). Les activités qui s'y déroulent sont souvent multiples, enchevêtrées et accomplies en coordination avec un grand nombre de partenaires de l'interaction (Garcia, Filliettaz, \& Wolter, à paraître ; Wolter, 2020). À ce titre, elles donnent lieu à un intense travail de coordination avec les parents et leurs enfants, à certains égards emblématique du travail interactionnel qu'engagent les professionnels dans le quotidien de leur activité.

Dans ce contexte particulier, cet article propose de porter une attention approfondie aux routines interactionnelles qui structurent le travail des éducatrices et des éducateurs à l'occasion des temps d'accueils des enfants dans la structure d'accueil, au moment où ils arrivent dans l'espace éducatif accompagnés de leurs parents. Il vise à mieux comprendre comment se coordonnent les participants au moment de ces accueils et les ressources qu'ils mobilisent dans le cadre de cette coordination. Comment les accueils sont-ils accomplis par les éducateurs, les enfants et leurs parents? Quelles récurrences et quelles variations sont repérables dans ces moments d'interactions? Quelles compétences interactionnelles sont mises en œuvre pour faire face aux contingences particulières de ces situations? Apporter des éléments de réponse à ces questions soulève d'importants défis sur le plan méthodologique. Comment définir une routine interactionnelle? Comment mettre en évidence des éléments récurrents et des variations dans l'analyse du travail ? Comment dépasser l'analyse de cas singuliers pour mettre en visibilité des réalités qui se construisent dans des temporalités longues et à travers une réitération de situations ? Dans le champ de l'analyse interactionnelle, ces questions ont récemment attiré l'attention d'un nombre croissant de chercheurs et la problématique de la quantification ne constitue plus une question mise de côté (Mondada, 2006, 2008; Schegloff, 1993; Stokoe, 2014). En continuité avec cette tendance, la démarche présentée ici vise aussi, plus généralement, 
à présenter et discuter des voies méthodologiques possibles permettant de rendre compte non seulement de la singularité des situations rencontrées, mais encore des routines qui permettent aux participants d'y faire face.

6 Pour ce faire, nous commençons par exposer, sur le plan théorique, la manière dont le caractère routinisé des rencontres sociales peut être conceptualisé dans une perspective interactionnelle en analyse du travail. Un dispositif empirique est alors présenté, qui a permis de documenter de manière méthodique des interactions verbales et non-verbales prenant place au moment des accueils et des départs des enfants et de leurs familles au sein de deux institutions de la petite enfance. Les temps d'accueil sont analysés dans leur dimension à la fois temporelle et multimodale, au moyen d'une méthodologie qui se déploie par étapes. Les situations d'arrivées sont d'abord caractérisées et quantifiées selon leur durée et les conditions de passage de l'enfant de la sphère parentale à la sphère institutionnel. Un cas singulier est ensuite analysé, puis mis en perspective avec d'autres occurrences impliquant des acteurs proches. Un cas contrasté est alors décrit, dans une occurrence singulière plus dans une série de situations. Enfin, dans une discussion conclusive, nous revenons sur les ingrédients des routines d'interaction et discutons leur rôle dans l'organisation du travail des professionnels de l'éducation de l'enfance. Nous abordons également comment la démarche d'analyse des interactions peut devenir une ressource pour la formation des éducatrices et éducateurs.

\section{Cadre théorique : une perspective interactionnelle sur les routines des rencontres sociales}

7 En référence aux travaux princeps d'Erving Goffman (1973a), l'interaction est ici définie comme une "rencontre sociale", ou plus précisément "le processus temporel et séquentiellement ordonné qui prend place lorsqu'au moins deux individus se trouvent dans un espace perceptuel partiellement partagé, dans lequel ils recourent à des ressources sémiotiques, en vue de conduire collectivement une action conjointe, ellemême indexée à des pratiques sociales historiquement et culturellement constituées " (Filliettaz, 2018). Ainsi définie, l'interaction peut être envisagée à la fois comme un objet et comme une centration de l'attention du chercheur qui suscite une méthode particulière de recherche. Elle constitue un objet de recherche, en ce que l'analyse porte de manière centrale sur les processus de coordination rendus visibles au moment où les participants s'engagent dans des formes d'interdépendance et d'intercompréhension pour faire face ensemble aux enjeux qui sous-tendent leurs rencontres sociales. Elle constitue aussi une méthode de recherche en analyse du travail en ce qu'elle conduit à privilégier un regard particulier sur ces processus de coordination dans l'action, qui les érige en principe d'intelligibilité et d'interprétation des comportements accomplis dans ces mêmes rencontres (Filliettaz, 2018).

8 En référence aux courants de la microsociologie de la vie quotidienne et à l'ethnométhodologie, les rencontres sociales conçues comme des interactions constituent des réalités à la fois ordonnées, ritualisées, et soumises à des jugements de conformité. Pour Garfinkel (2007), fondateur de l'ethnométhodologie, l'ordre social apparait comme une "méthode» produite par les membres d'une communauté au moment de faire face aux problèmes pratiques que suscite leur rencontre. Pour résoudre ces problèmes pratiques, les membres font advenir par leurs comportements 
un ordre reconnaissable et auquel les partenaires de l'interaction peuvent s'aligner ou non. Les rituels qui ponctuent les interactions (Goffman, 1974), comme par exemple les échanges confirmatifs d'ouvertures et de clôtures des rencontres sociales, constituent des exemples emblématiques de telles "méthodes", par lesquelles les participants se signalent mutuellement la manière dont ils se repèrent dans la progression de leur rencontre et plus généralement leur appartenance à la communauté des individus qui partagent ces manières de faire. Aussi les conditions de participation des individus à des interactions produisent-elles des attentes relatives aux lignes de conduites attendues et reconnues comme légitimes dans les situations concernées. Ces lignes de conduite sont relatives à la nature des activités en cours et aux enjeux qui les soustendent. Elles sont aussi imputables aux rôles respectifs des participants à l'égard de ces activités. C'est en effet au regard de ces rôles et de ces enjeux que les contributions respectives des participants sont évaluées et que sont mises en jeu leurs rapports de faces (Goffman, 1973a ; Bucholtz \& Hall, 2005).

9 Les principes organisateurs des rencontres sociales sont non seulement rendus manifestes à travers la ligne de conduite des participants, mais ils sont aussi négociables dans les conditions singulières dans lesquelles ils sont actualisés. A cet égard, on doit considérer que les interactions sociales procèdent d'une double logique, à la fois schématique et émergente, comme le relève ici Bange (1992): "Toute interaction a pour condition de réaliser un schéma fonctionnel qui appartient au savoir commun des partenaires : c'est son côté schématique ; elle le fait dans des formes non prédictibles, car dépendantes de la relation qui se noue entre des individus dont chaque histoire est singulière : c'est son côté émergent. » (p. 211). Ces différents ingrédients de l'ordre de l'interaction méritent d'être précisés.

\subsection{La composante schématique de l'ordre de l'interaction}

Si l'accomplissement de l'action présente un caractère irrémédiablement social, c'est non seulement parce qu'elle est susceptible d'être évaluée par autrui, mais c'est surtout parce qu'elle procède d'une signification qui lui est conférée par des entités qui dépassent la sphère d'influence des individus. Dans la perspective phénoménologique sociale développée dans les travaux d'Alfred Schütz, ces principes s'incarnent dans les concepts de typicalité et d'intersubjectivité. Schütz (1987) décrit au moyen de trois étapes les conditions psycho-sociales liées à l'accomplissement des actions. Dans le cadre de leurs conduites finalisées, les individus ont accès à des connaissances liées à leurs expériences passées et ils peuvent par conséquent partiellement orienter leurs actions présentes au moyen d'une forme de typicalité qu'elles partagent avec les actions passées : "Tous les projets de mes actes à venir sont basés sur la connaissance dont je dispose au moment de l'élaboration du projet. C'est mon expérience d'actes menés auparavant, similaires dans leur typicalité au projet, qui vient nourrir cette connaissance. » (Schütz 1987, p. 27). Ces typifications intervenant dans un monde social, dominé par des rapports interindividuels, il faut considérer dans un second temps qu'au-delà des déterminations biographiques propres à chaque individu, ces expériences présentent un certain degré de ressemblance d'un individu à l'autre et que par conséquent, elles sont partagées intersubjectivement. C'est précisément sur la base de ce caractère intersubjectif des préexpériences typifiantes que les individus sont conduits à développer ce que Schütz appelle des autotypifications de leurs propres conduites. C'est en observant que de façon régulière des événements se produisent et 
en admettant que ces événements sont susceptibles d'arriver à d'autres que les individus adoptent des lignes de conduite alignées et ajustées à ces attentes sociales.

11 C'est très explicitement dans la continuité des travaux d'Afred Schütz qu'Erving Goffman inscrit sa réflexion sur l'action lorsqu'en 1974, il publie Frame analysis, publié ultérieurement en français sous le titre Les cadres de l'expérience (Goffman, 1991). Dans sa théorie du cadrage de l'expérience, Goffman soutient que la manière dont les individus font l'expérience des réalités qu'ils rencontrent dans la vie quotidienne n'est pas immédiate et transparente, mais médiatisée, par des "prémisses organisationnelles" ce qu'il appelle des cadres (frames). Ces cadres désignent un ensemble de savoirs et de savoir-faire, culturellement construits, qui permettent d'interpréter les expériences à la fois naturelles et sociales comme relevant d'un type particulier, et de répondre à la question «qu'est-ce qui se passe ici?». C'est notamment en mobilisant ces cadres naturels et sociaux que les individus parviennent à interpréter la signification des situations qu'ils rencontrent et d'y ajuster leurs propres comportements. Si ces cadres jouissent, sur le plan sociétal, d'une forme de réalité partagée par les membres d'une communauté, leur convocation en situation constitue une opération à chaque fois nouvelle et le produit d'un travail d'interprétation incessant. C'est en ceci que réside, pour Goffman, le caractère "vulnérable » et nécessairement incertain du cadrage de l'expérience. Les individus peuvent se tromper sur la signification de ce qu'ils font. Ils peuvent être amenés à reconsidérer rétrospectivement la manière d'interpréter une expérience vécue. Ou encore, ils peuvent détourner ou renégocier les attentes sociales qui régissent l'activité en cours. Ici aussi, la dynamique propre aux interactions constitue une des ressources par lesquelles des contextes locaux d'activité peuvent être collectivement établis et interprétés. Ceci nous amène à considérer la composante émergente de l'ordre de l'interaction.

\subsection{La composante émergente de l'ordre de l'interaction}

12 Si l'action donne lieu à des typifications et peut faire l'objet de cadrages, elle n'en procède pas moins d'un rapport indexical à des situations singulières, nécessairement rapportables aux contingences locales rencontrées par les participants à l'interaction. C'est là une facette de la problématique de l'action et de ses conditions d'interprétation qui a été fortement investie par le tenant du courant de la cognition dite située ou distribuée (Relieu, Salembier, \& Theureau, 2004), en référence en particulier à la théorie ethnométhodologique de l'action (Suchman, 1987). Selon cette perspective, les actions, bien que présentant un caractère ordonné, ne sont jamais planifiées dans le sens fort que la psychologie cognitive attribue aux concepts de scénario ou de scripts (Schank \& Abelson, 1977 ; von Cranach, Kalbermatten, Indermühle, \& Gugler, 1982). Les plans sont plutôt considérés comme une ressource que les acteurs construisent et consultent avant et après l'accomplissement de l'action. Pour Suchman (1987, p. ix), ce n'est que lorsque les individus sont contraints de rendre compte de la rationalité de leurs actions qu'ils invoquent l'orientation d'un plan. Énoncés à l'avance, les plans sont nécessairement vagues, dans la mesure où ils doivent s'adapter aux imprévus de situations particulières. Reconstruits a posteriori, les plans filtrent systématiquement la particularité des détails qui caractérisent les actions situées, au profit des aspects des actions qui peuvent être considérés comme conformes aux plans. L'alternative proposée consiste à fonder les conduites des participants sur la capacité de ces derniers 
à s'orienter dans une situation déterminée et à s'adapter en permanence aux paramètres de l'environnement social. Ainsi, les ressources de l'agir résident moins dans des plans prédéfinis que dans la possibilité pour les participants de développer une " sensibilité aux circonstances locales » (Suchman 1987, p. 27). Selon cette logique, le caractère «situé » de l'action prime sur les structures mentales qui en déterminent l'organisation dans un modèle de la planification.

Cette manière d'envisager la place des processus de planification dans les conditions d'accomplissement des rencontres sociales conduit à une révision profonde dans la conceptualisation des relations entre les actions et les intentions qui les sous-tendent. Plutôt que de concevoir ces relations comme procédant d'un dualisme entre des domaines de réalité ontologiquement distincts - des conduites observables d'une part, et des ressources cognitives d'autre part - il s'agit au contraire de considérer comme des «relations internes" les rapports de l'intention et de l'action, du sujet et de ses actions, de l'action et du discours de l'action : «Dans une telle perspective, c'est dans l'action effective et publique que l'intention trouve son expression naturelle. [...] L'intention n'est plus alors l'état mental indépendant que distingue le discours de l'action : elle appartient entièrement à l'action accomplie, elle est incorporée en elle " (Quéré, 1990, p. 95). Et cette intention n'est pas réductible à l'action des sujets. Elle requiert de la part des partenaires de l'interaction plus qu'une simple constatation, mais une appropriation, une « opération de configuration qui prolonge et achève celle de l'agent» (ibid., p.103), conférant ainsi à l'intention-dans-l'action un caractère intrinsèquement social.

Cette manière de concevoir la part émergente de l'ordre de l'interaction n'est pas sans conséquence sur les conditions pratiques dans lesquelles sont attribuées des intentions aux conduites rendues manifestes dans l'action. Dès lors, les intentions-dans-l'action ne sont pas seulement inhérentes aux sujets agissants, mais elles prennent forme dans les modalités d'évaluation manifestées par les co-participants à l'interaction. À cet égard, elles ne sont pas repérables dans les comportements eux-mêmes, mais dans leurs conditions d'enchainement dans le temps. C'est là une facette de la problématique de l'action dans l'interaction qui a été explicitement théorisée par les pionniers de l'analyse conversationnelle, pour qui l'organisation séquentielle de l'interaction constitue un exemple emblématique d'ordre méthodique produit par les membres pour résoudre les problèmes pratiques soulevés par la problématique de la coordination dans l'action (Sacks, 1992 ; Sacks, Schegloff, \& Jefferson, 1978 ; Schegloff, 2007). Ainsi donc, le déploiement de l'action étape par étape constitue-t-il une matrice dans laquelle s'inscrit la signification et l'organisation de l'action, entendue comme un accomplissement intersubjectif.

Il serait réducteur cependant de considérer que la signification de l'action séquentiellement et collectivement construite dans les enchainements des tours d'action repose exclusivement sur des prises de paroles et donc des ingrédients de nature langagière. Si l'on admet que «l'habitat naturel de la parole est un lieu où la parole n'est pas toujours présente ", alors il faut reconnaître aussi que "l'étude du comportement verbal nous entraine plaisamment vers l'étude du comportement sans parole» (Goffman, 1988, p. 145). En continuité avec les approches multimodales en analyse du discours et de l'interaction (Goodwin, 2000; Kress, Jewitt, Ognorn, \& Charalampos, 2001; Mondada, 2017), il est admis que les significations en circulation dans l'interaction sont fondées, non plus seulement sur le substrat verbal de 
l'interaction, mais sur une large palette de ressources (parole, prosodie, gestes, postures corporelles, objets matériels, pratiques scripturales, etc.). Ainsi donc, dans le cadre de ces processus dynamiques de création et d'attribution de signification, les participants à l'interaction recourent à des ressources diverses, qui constituent des systèmes sémiotiques variés ou des modes (Kress et al., 2001). La signification construite en contexte procède rarement de la mobilisation d'un mode unique. Elle repose au contraire fréquemment sur des agrégations et des combinaisons de modes, convoquées simultanément selon les potentialités qui leur sont propres. Par exemple, le recours à la parole rend possible la convocation de contenus abstraits, alors que le pointage sur un objet matériel permet son repérage visuel. Plutôt que de procéder d'une alternance entre modes convoqués successivement, la création de la signification repose sur une articulation multimodale et contingente de la situation créée. C'est donc aussi sur cette combinaison et cette imbrication de ressources en contexte que repose la composante émergente de l'ordre de l'interaction.

\section{Cadre méthodologique : documenter les arrivées et les départs des enfants dans les structures d'accueil}

16 Les rencontres entre les éducatrices et éducateurs et les parents se déroulent principalement lors des arrivées et départs quotidiens, ainsi que pendant les entretiens de bilans. Afin de pouvoir étudier le travail des éducatrices et éducateurs en présence des parents, une démarche d'enquête à caractère vidéo-ethnographique a été conduite entre le printemps 2018 et le printemps 2019 au sein de deux institutions de la petite enfance en Ville de Genève (Suisse). Sur la base d'un partenariat conclu avec les institutions et un accord formel des professionnels et des parents, une observation filmique a été réalisée, durant deux semaines consécutives, au sein des structures d'accueil. Par ailleurs, un dispositif de formation continue a été implémenté dans chacune des structures d'accueil sur la base d'une analyse des films enregistrés et en mobilisant les apports de l'analyse des interactions. Lors des séances d'analyse, les éducatrices ont pu apporter leur point de vue sur les situations filmées, décrire les conduites observables des différents interactants dans les films et échanger avec l'équipe de recherche concernant leurs pratiques. Ce volet du programme de recherche n'est pas abordé dans cette contribution et donne lieu à des travaux spécifiques publiés par ailleurs (Garcia, 2021a, 2021b ; Garcia \& Filliettaz, 2020).

Pour le recueil des données portant sur les arrivées et les départs, il s'agit de perturber le moins possible les situations authentiques. Ainsi, les enregistrements vidéo ont été effectués par des caméras fixes, installées pendant la durée des arrivées ( $7 \mathrm{~h} 30-9 \mathrm{~h} 30)$ et des départs ( $17 \mathrm{~h} 30-19$ h 00), et ce dans différents groupes d'enfants. Une pluralité de prises de vue a permis d'assurer une large couverture du champ d'action des participants. Le tableau 1 ci-dessous retrace le nombre de jours, les groupes d'enfants concernés et la durée totale des films réalisés. Il indique que 38 contextes d'arrivées ou de départs ont pu être observés dans deux groupes d'âge du site I, et 65 contextes relatifs à quatre groupes d'âge dans le site II. Au total, 178 heures d'enregistrements ont été effectuées. 
Tableau 1 : Recueil des données brutes. Table 1: Collection of raw data

\begin{tabular}{l|l|l|l|l|}
\hline \multicolumn{6}{l|}{ Arrivées et départs } \\
\hline Site & $\begin{array}{l}\mathrm{N}^{\circ} \text { de } \\
\text { jours }\end{array}$ & Groupes & $\begin{array}{l}\text { Total contextes } \\
\text { filmés }\end{array}$ & $\begin{array}{l}\text { Total durée des } \\
\text { films }\end{array}$ \\
\hline Site I & 10 & 2 groupes (bébés, petits) & 38 contextes & 76.02 heures \\
\hline Site II & 12 & 4 groupes (bébés, petits, moyens, grands) & 65 contextes & 102.32 heures \\
\hline
\end{tabular}

Le nombre de participants filmés se chiffre au total à 248 participants. Dans le Site I, il s'agit de 44 enfants et de leurs parents ainsi que de 16 professionnel.le.s, tandis que dans le Site II, 61 enfants et leurs parents et 22 professionnel.le.s apparaissent dans les films. Les données brutes ont été traitées et analysées au moyen du logiciel Transana MU, spécialisé dans l'étude qualitative des données interactionnelles audio-vidéo (https://www.transana.com). À l'aide de ce logiciel, les données filmiques brutes ont été organisées dans une base de données et structurées selon une logique de site et de groupe d'âge des enfants. Étant donné que les caméras fixes filment en continu, une première étape de traitement des données a consisté à identifier, au sein des données brutes, les séquences d'arrivées et de départs pour chacun des participants filmés. Pour ce faire, une démarche de codage temporel a été menée sur l'ensemble des films monté sur la base de données. Chaque séquence d'arrivée ou de départ a été identifiée à l'aide d'un code temporel marquant le début et la fin de la séquence, ainsi que par l'identification des participants impliqués. La durée des séquences est délimitée par la présence des parents dans le champ visuel des caméras. Le début de la séquence est marqué lorsque le parent ouvre la porte de la salle ou apparaît à l'entrée de la salle. La séquence prend fin lorsque le parent ferme la porte de la salle ou sort de la salle. Afin de faciliter l'anonymisation des participants, l'identification des participants se fait par un code qui différencie les enfants, les parents et les professionnel.le.s.

Figure 1 : Repérage des séquences sur le logiciel de Transana MU. Figure 1: Locating the sequences on the Transana MU software

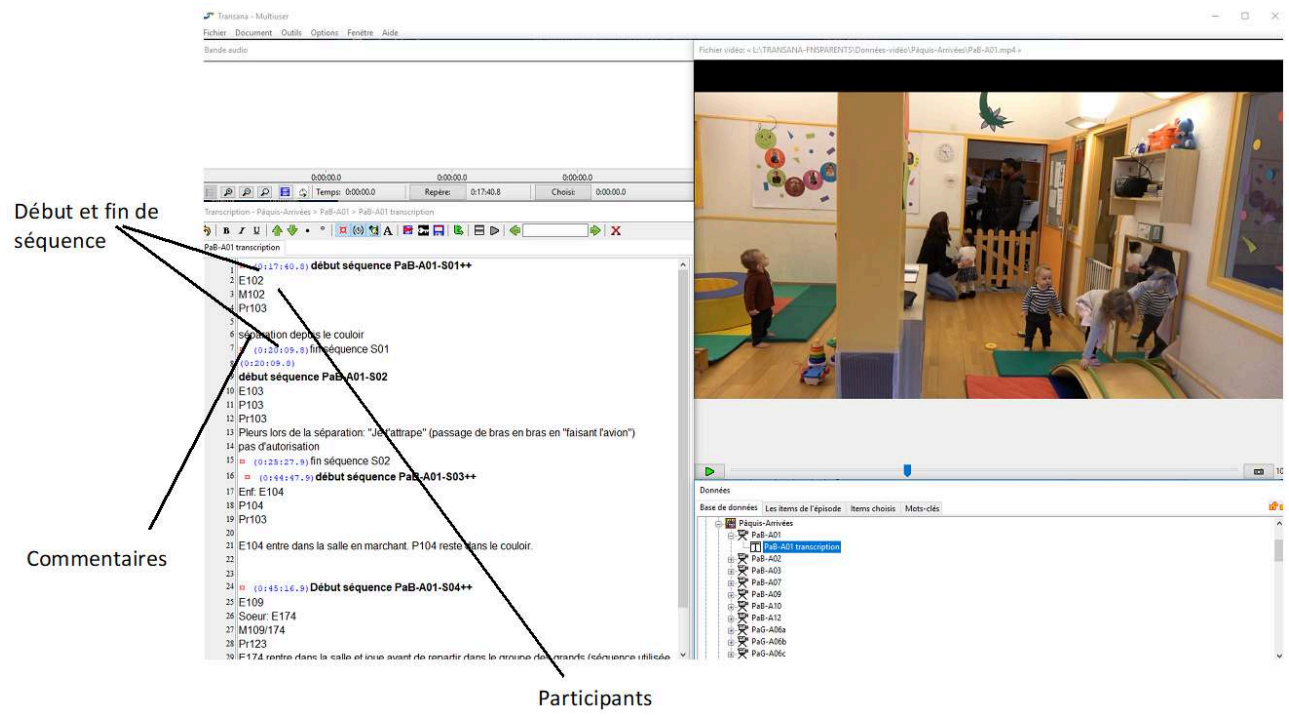

Ce travail de codage temporel des données brutes a permis de repérer le nombre et la durée des séquences d'arrivées et de départs dans l'ensemble des films disponibles. Dans le Site II, retenu pour l'étude présentée dans cet article, 335 séquences d'arrivées 
et 314 séquences de départs au total ont été identifiées. S'agissant des accueils, 18 arrivées ont été repérées pour le groupe des Bébés, 102 pour le groupe des Petits, 113 pour le groupe des Moyens et 102 pour le groupe des Grands, le tout pour un total de 10 heures et 25 minutes.

Tableau 2 : Recueil des séquences d'arrivées et de départs pour le site II. Table 2: Collection of arrival and departure sequences for Site II

\begin{tabular}{|c|c|c|c|c|c|c|c|c|c|c|}
\hline \multirow[t]{3}{*}{ Situation } & \multicolumn{8}{|c|}{ Groupe } & \multirow{2}{*}{\multicolumn{2}{|c|}{ Total groupes }} \\
\hline & \multicolumn{2}{|c|}{ Bébés } & \multicolumn{2}{|c|}{ Petits } & \multicolumn{2}{|c|}{ Moyens } & \multicolumn{2}{|c|}{ Grands } & & \\
\hline & No & Durée & No & Durée & No & Durée & No & Durée & No & Durée \\
\hline Arrivées & 18 & 1.09 .49 & 102 & 2.53 .36 & 113 & 2.56 .36 & 102 & 3.24 .28 & 335 & 10.25 .16 \\
\hline Départs & 39 & 2.55 .19 & 106 & 6.45 .38 & 59 & 2.00 .11 & 110 & 6.58 .55 & 314 & 18.40 .03 \\
\hline
\end{tabular}

\section{Cadre analytique : L'accueil des enfants comme routine interactionnelle}

Dans les paragraphes qui suivent, nous présentons une démarche analytique qui permet de cerner progressivement le caractère routinier des activités prenant place au moment des accueils des enfants sur le site II. Pour ce faire, une combinaison d'observations quantitatives et qualitatives est proposée. L'élaboration de ces démarches méthodologiques s'est construite par des tâtonnements successifs qui peuvent être synthétisés en cinq étapes. La première étape consiste à catégoriser les séquences d'arrivées selon leur durée et différentes variables observables dans le positionnement spatial de l'enfant (4.1.). Dans un deuxième temps, l'analyse vise à décrire, au moyen d'une étude de cas, un déroulement particulier d'accueil au moment où l'enfant est passé de bras en bras entre le parent et l'éducateur (4.2.). D'autres séquences concernant ce même enfant sont alors comparées, dans le but de faire ressortir des éléments de récurrence et de variation (4.3.). Pour suivre, une situation d'accueil contrastée concernant un autre enfant est décrite, d'abord sous la forme d'un cas unique (4.4.) puis en contraste avec une série d'autres occurrences observées dans les données disponibles (4.5.).

\subsection{Catégoriser les séquences selon leur durée et le positionnement de l'enfant}

21 Le bornage temporel effectué pour identifier les séquences portant sur des arrivées et des départs oriente vers la rapidité ou la lenteur des rencontres entre les parents, les enfants et les professionnelles. Afin de pouvoir étudier les propriétés temporelles de ces rencontres "sur le pas-de-porte », la durée des séquences a été mesurée. Le logiciel Transana permet de constituer des extraits (clips) à partir des codes temporels saisis dans les transcriptions. Un extrait peut être défini comme un segment de film et sa transcription borné par deux codes temporels. Une fois constitué, un extrait permet de mesurer de manière automatique la durée de la séquence étudiée. Cette démarche de repérage d'extraits a été effectuée systématiquement sur l'ensemble des séquences 
d'arrivées du Site II, soient 335 occurrences. Elle permet de mesurer les durées moyennes des accueils, selon les groupes d'âge concernés.

Figure 2 : Durée des séquences d'arrivées (Site II).

Figure 2: Duration of arrival sequences (Site II)

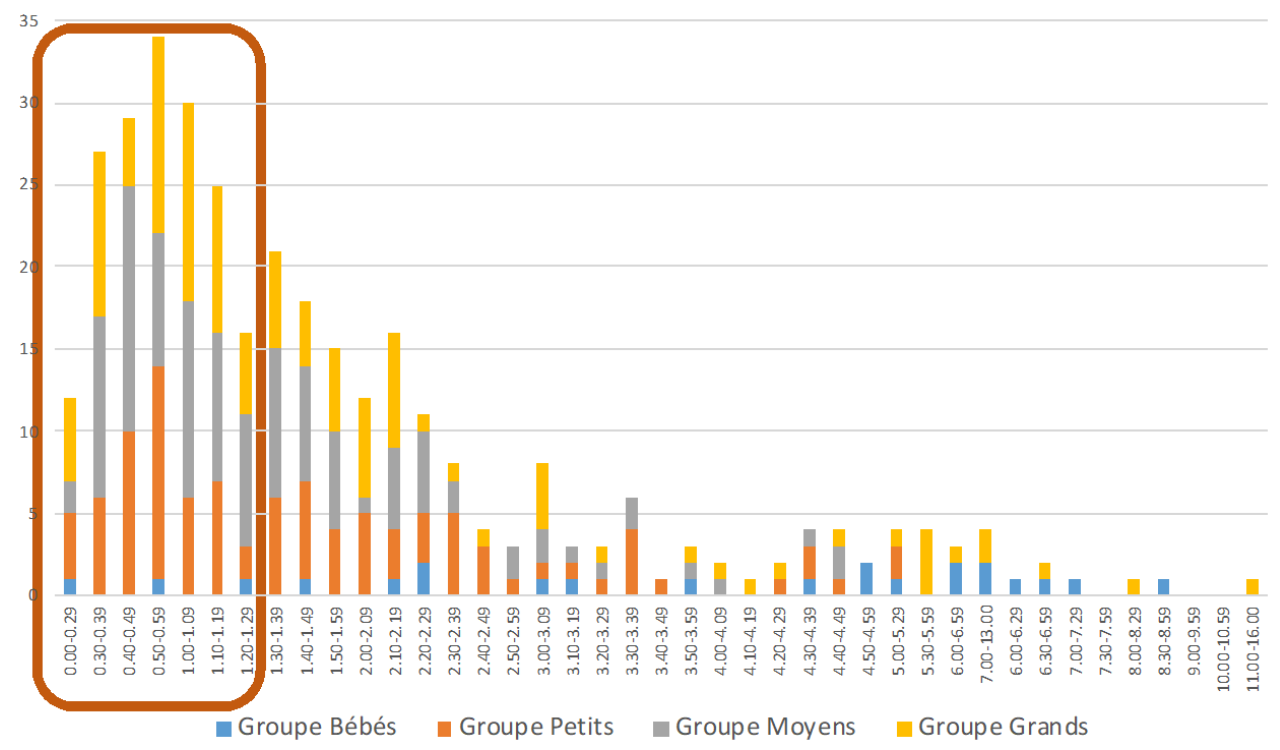

Il ressort de la Figure présentée ci-dessus que $50 \%$ des séquences d'arrivées observées sur le Site II se situent entre 0:00 et 1:29 minutes. Le nombre d'extraits d'arrivées le plus élevé se situe dans la tranche de 50 à 59 secondes et comporte 35 occurrences. Ces résultats mettent en évidence la relative brièveté des échanges qui interviennent à l'occasion des rencontres entre les parents et les éducateurs au moment des accueils.

En tenant compte des difficultés d'exploiter certaines séquences liées aux limites du champ de la caméra, aux bruits ambiants ou aux autorisations manquantes, la démarche de confection d'extraits sur Transana conduit à la constitution d'une collection de 72 séquences exploitables, d'une durée de moins de 1:09 minutes. Ce premier repérage permet de s'intéresser aux ingrédients qui favorisent la rapidité des arrivées. À ce propos, l'identification de plusieurs centaines de séquences impliquant un grand nombre de participants s'effectue à travers un «balayage » du regard porté sur les films vidéo. Pour situer les débuts et les fins des séquences, le regard s'oriente notamment vers l'entrée de la salle pour voir apparaître et disparaître les parents du champ de la caméra. Le repérage des séquences amène à une focalisation sur les phénomènes interactionnels visibles et se centre ainsi moins sur le langage verbal, à ce stade de l'analyse. Le regard se porte notamment sur le positionnement des enfants au moment de leur arrivée dans la structure d'accueil. Selon leur âge et leurs besoins spécifiques, les enfants peuvent entrer dans l'espace éducatif soit en marchant, soit porté par leur parent. Ils peuvent également être passés de bras en bras entre les parents et les éducateurs, ou rejoindre l'éducateur en marchant. Selon cette observation, quatre configurations à caractère spatial émergent de l'analyse: 1) l'enfant arrive en marchant et entre dans la salle en marchant (Enfant marche \& marche - M/M) ; 2) l'enfant arrive en marchant et, lors du passage, le parent le prend dans les bras pour le passer à l'éducatrice ou à l'éducateur (Enfant marche \& porté - M/ P) ; 3) l'enfant arrive en étant porté dans les bras de son parent, qui le pose par terre pour qu'il entre en marchant dans la salle (Enfant porté \& marche - P/M) ; 4) l'enfant 
arrive en étant porté par son parent et il est passé de bras en bras (Enfant porté \& porté $-\mathrm{P} / \mathrm{P})$.

24 À partir de ces deux critères d'analyses, liés à la temporalité d'une part et au positionnement spatial de l'enfant d'autre part, il devient possible de classifier les extraits contenus dans la collection. L'analyse d'un cas singulier vise ensuite à étudier quels liens peuvent être identifiés entre la temporalité d'un extrait et le positionnement de l'enfant. Comme point de départ, le choix s'est porté sur un extrait de courte durée impliquant un enfant qui arrive en étant porté par sa mère, qui le passe dans les bras de l'éducatrice.

\subsection{L'analyse d'un cas singulier : l'accueil d'Alex}

La séquence présentée ci-dessous se déroule à la fin d'une période d'accueil. La plupart des enfants sont déjà présents lorsque Alex (A) arrive avec sa mère (MA). Les deux professionnelles du groupe, $\mathrm{E}$ et $\mathrm{C}$ (pour les conventions de transcriptions, voir annexe I), sont en train de regrouper les enfants pour une nouvelle activité. E continue à s'occuper encore un petit moment des enfants, avant de s'orienter et de s'approcher d'Alex et de sa mère. La séquence dans son ensemble dure 1:10 minutes. L'extrait analysé ci-dessous dure 20 secondes et il est délimité par l'approche de l'éducatrice d'Alex et de sa mère jusqu'au départ de la mère.

Figure 3 : Durée de l'extrait 1.

Figure 3: Duration of extract 1

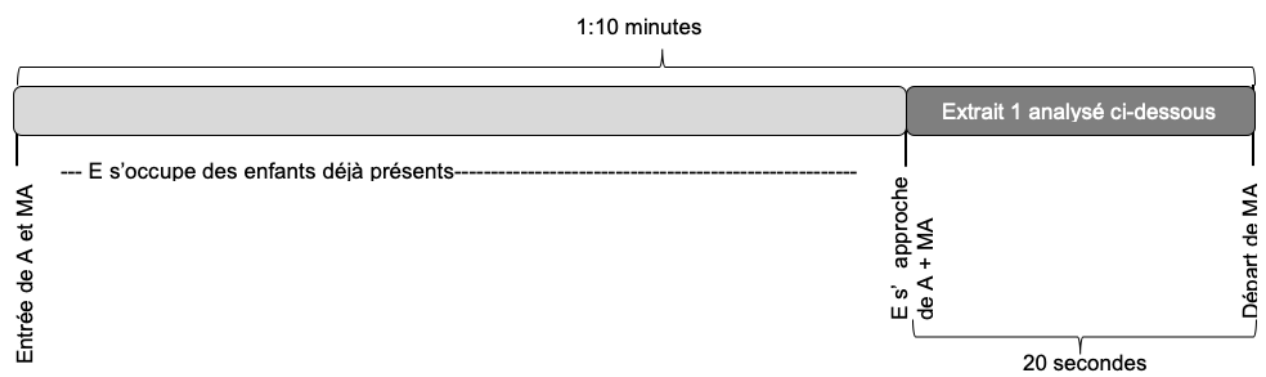

$\mathrm{Au}$ début de cet extrait 1, l'éducatrice $\mathrm{E}$ s'approche d'Alex et de sa mère. La mère porte Alex dans ses bras et elle est positionnée au milieu de la salle de jeux des enfants. 
Extrait 1 (A) : ouverture de l'interaction

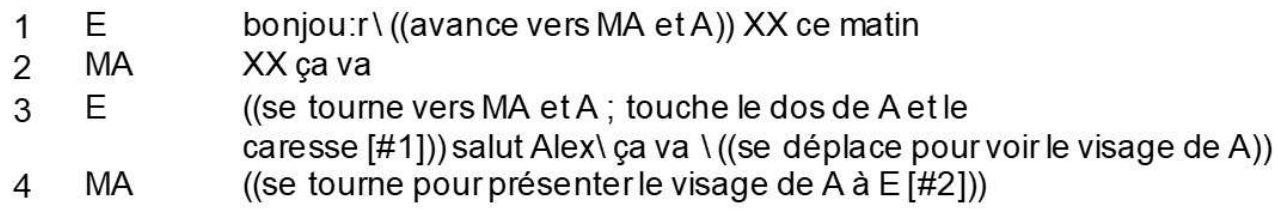

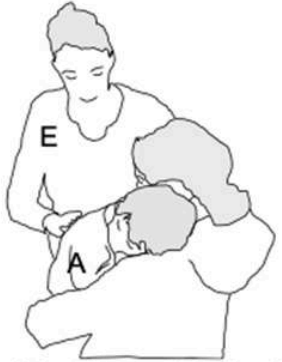

\#1 : E touche le dos de A

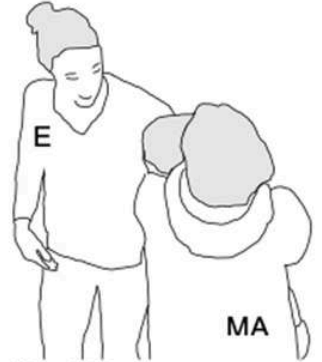

\#2: MA se tourne pour présenter le visage de $\mathrm{A}$ à $\mathrm{E}$ d'ouvertures assimilables à un échange confirmatif au sens de Goffman (1973b) (l.1). Ces salutations sont adressées simultanément à la mère et à l'enfant. La mère y répond (1. 2) ; Alex est lové contre l'épaule de sa mère et n'esquisse pas de mouvement lors de l'arrivée de l'éducatrice. $\mathrm{E}$ touche alors le dos d'Alex avec une légère caresse (voir \#1). Elle s'adresse spécifiquement à l'enfant en le saluant encore une fois et se déplace pour pouvoir observer le visage d'Alex. Simultanément, la mère d'Alex se déplace également pour présenter le visage de son enfant à $\mathrm{E}$ (voir \#2). Ce repositionnement des participants rend possible un échange de regards direct entre l'éducatrice $\mathrm{E}$ et Alex, qui reste toujours lové dans le cou de sa mère.

Cette ouverture de l'interaction montre comment l'éducatrice et la mère s'ajustent mutuellement afin de permettre une prise de contact entre Alex et $\mathrm{E}$. Les salutations s'accompagnent d'un alignement des corps et des regards, de sorte à créer un espace d'interaction entre la professionnelle et l'enfant accueilli.

Suite aux salutations et dès que l'éducatrice a établi un contact visuel avec Alex, elle lui présente ses deux mains, paumes ouvertes et dressées vers le haut (voir \#3): 

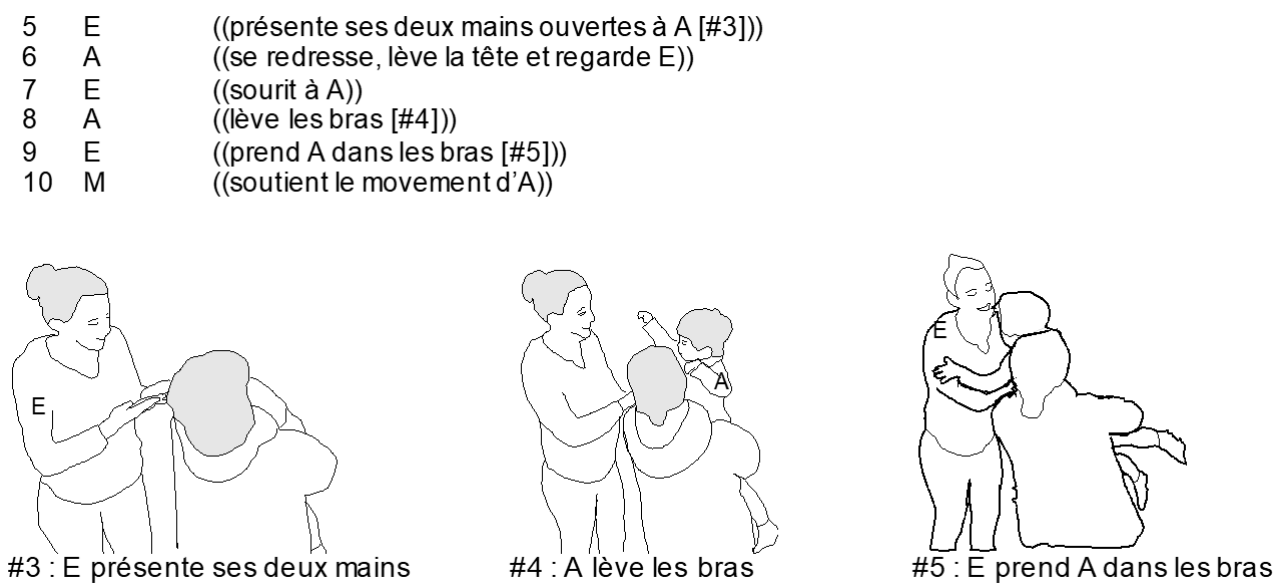

Le geste de l'éducatrice agit ici comme un geste d'invitation. Il déclenche immédiatement une réponse d'Alex et de sa mère. De manière séquentiellement ordonnée, l'enfant se redresse, tend ses bras, et s'oriente vers $\mathrm{E}$, rendant par là manifeste qu'il a interprété le geste de l'éducatrice comme une action d'invitation à être pris dans les bras. La mère soutient le mouvement de l'enfant et continue à soutenir son poids, pendant que E prend Alex dans les bras. Elle aussi s'oriente vers l'action de passage, initiée par $\mathrm{E}$ et dans laquelle Alex a commencé à s'engager. Le geste d'invitation est donc suivi par une coordination fine des trois interactants pour effectuer ensemble un mouvement rapide et fluide. L'éducatrice, Alex et sa mère s'alignent pour faciliter le passage des bras de la mère aux bras de E. L'enfant n'est pas ici « passé » comme une sorte d'objet de l'une à l'autre, mais participe activement à un mouvement collectivement accompli. 


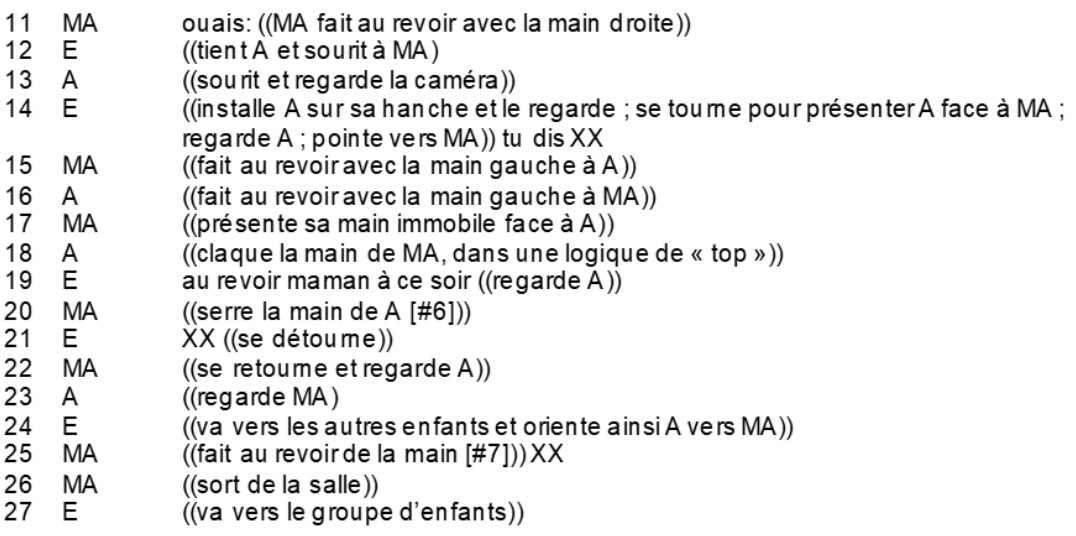

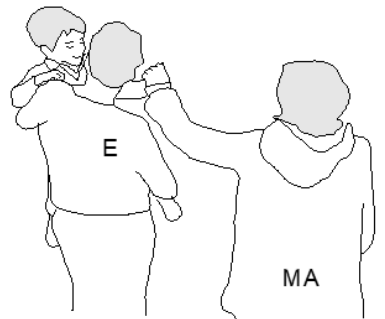

\#6 : MA serre la main de $A$

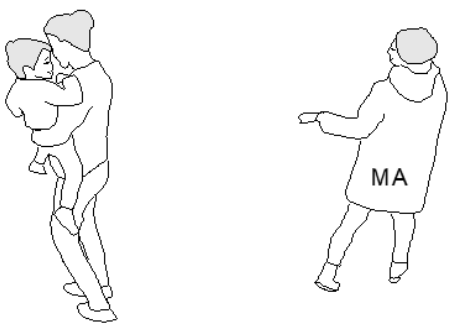

\#7 : A regarde MA et MA fait au revoir de la main

31 La mère d'Alex ratifie le passage rapide d'Alex dans les bras de l'éducatrice par un « ouais : ( (l.11) et enchaîne immédiatement en faisant un geste d'au revoir de sa main droite. Pendant l'action de portage d'Alex dans ses bras, l'éducatrice sourit de manière continue, même une fois Alex passé dans ses bras (1.12). Elle regarde ensuite l'enfant, se tourne pour le présenter face à sa mère, pointe vers elle et demande à Alex de dire au revoir à sa mère (1.14). La mère présente alors sa main à Alex (1.17), qui répond à ce geste par un «top » de la main, montrant par là qu'il a bien compris l'invitation de la mère d'accomplir un échange gestuel de salutation. Le geste co-construit par Alex et sa mère présente ici une signification quasi-linguistique et fonctionne comme un " emblème " (McNeill, 2000). E accompagne ce geste d'au revoir d'Alex et de sa mère en prenant la parole à la place de l'enfant : « au revoir maman à ce soir» (1. 19). Elle rend ainsi manifeste la signification qu'elle attribue au geste de l'enfant. La mère serre ensuite la main d'Alex (\#6). Dès la fin de cet échange entre Alex et sa mère, l'éducatrice se détourne et dit au revoir à la mère. En s'éloignant vers les autres enfants, E se positionne de dos à la mère. Ceci permet cependant à Alex de regarder encore une fois sa mère qui lui fait un dernier geste d'au revoir à distance (\#7), avant de sortir de la salle.

L'analyse de cet accueil permet de mieux comprendre en quoi résident la fluidité et la brièveté de l'arrivée d'Alex. Malgré le peu de moyens langagiers déployés, les actions accomplies par les participants s'enchainent rapidement et se répondent dans un alignement séquentiel clairement repérable. Avant le passage d'Alex dans les bras de l'éducatrice, des orientations corporelles et des gestes d'invitation permettent de configurer un moment pertinent permettant à l'enfant de changer de position dans l'espace. Après le passage d'Alex, des échanges répétés de regards, de gestes et des salutations permettent à chacun des participants de s'orienter progressivement vers 
une clôture de l'interaction et vers le départ de la mère. De cette manière, il devient possible de vérifier l'alignement de chacun des participants, et notamment d'Alex, au déroulement de ce moment d'accueil. On peut se demander si les conduites ordonnées mises en évidence dans cette étude de cas se rapportent aux contingences spécifiques de la situation rencontrée ou si elles présentent une validité qui dépasse les caractéristiques de cette seule situation. Comment Alex est-il accueilli à d'autres occasions? Qu'advient-il lorsqu'il est accueilli par une autre éducatrice ou accompagné par d'autres membres de sa famille? Les conduites des participants observées dans cet accueil singulier sont-elles susceptibles de se répéter ou diffèrent-elles d'une situation à l'autre ? Ce sont ces questions que nous proposons d'examiner ci-dessous.

\subsection{L'analyse d'une trajectoire : les accueils d'Alex}

Afin d'étudier si la situation d'arrivée présentée ci-dessus contient des éléments récurrents, l'analyse porte ici sur l'ensemble des arrivées d'Alex telles qu'elles dessinent une trajectoire d'accueil de l'enfant au fil du temps. Dans l'ensemble des données filmiques disponibles, il est possible d'identifier quatre situations d'arrivées exploitables concernant Alex. La première de ces séquences a été analysée ci-dessus [Extrait 1(A)] et elle est remobilisée dans l'analyse de cette trajectoire dans le but de faciliter les comparaisons. Dans chacune de quatre séquences concernant Alex, celui-ci arrive en étant porté dans les bras de son parent, respectivement sa mère ou son père. Dans un cas comme dans l'autre, il est toujours passé de bras en bras. Le positionnement d'Alex est dès lors identique dans l'ensemble des séquences qui le concernent et correspond à la catégorie " Enfant porté \& porté $(\mathrm{P} / \mathrm{P})$ ».

Les séquences d'arrivées d'Alex varient fortement en fonction du moment de l'arrivée, de l'espace et des participants concernés. Le premier extrait (analysé ci-dessus) se déroule à la fin de la matinée, dans l'espace habituel du groupe d'Alex et en présence de sa mère et de l'éducatrice de référence de son groupe. L'extrait 2 se déroule au début de la période d'accueil, dans le même espace et avec la même éducatrice, mais en présence du père d'Alex. Le père est également présent dans les extraits 3 et 4 . L'extrait 3 se passe en fin de matinée, dans une autre salle et avec une autre éducatrice. L'extrait 4 se déroule en milieu de matinée, dans la salle habituelle, et avec une éducatrice remplaçante. Mais ces éléments de variation situationnelle ne semblent pas exercer une influence sur le positionnement spatial de l'enfant, ni même sur la durée des accueils, comme le montre le tableau ci-dessous :

Tableau 3 : les contextes des séquences d'arrivées d'Alex.

Table 3: Contexts of Alex's arrival sequences

\begin{tabular}{|c|c|c|c|c|c|c|c|c|c|c|c|}
\hline \multirow{2}{*}{ 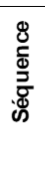 } & \multicolumn{3}{|c|}{ Moment de la matinée } & \multicolumn{2}{|c|}{ Espace } & \multicolumn{2}{|c|}{ Parent présent } & \multicolumn{2}{|c|}{ Professionnelle } & \multicolumn{2}{|l|}{ Durée } \\
\hline & Début & Milieu & Fin & $\begin{array}{l}\text { Espace } \\
\text { habituel }\end{array}$ & $\begin{array}{l}\text { Autre } \\
\text { espace }\end{array}$ & Mère & Père & $\begin{array}{l}\text { Educa- } \\
\text { trice du } \\
\text { groupe }\end{array}$ & $\begin{array}{l}\text { Autre } \\
\text { éduca- } \\
\text { trice }\end{array}$ & $\begin{array}{l}\text { Séquence } \\
\text { totale }\end{array}$ & $\begin{array}{l}\text { Durée } \\
\text { Extrait }\end{array}$ \\
\hline 1 & & & & & & & & & & $1: 10$ & $0: 20$ \\
\hline 2 & & & & & & & & & & $0: 51$ & $0: 24$ \\
\hline 3 & & & & & & & & & & $0: 26$ & $0: 16$ \\
\hline 4 & & & & & & & & & & $0: 50$ & $0: 22$ \\
\hline
\end{tabular}

En effet, indépendamment de ces variations situationnelles, les arrivées d'Alex se caractérisent par une grande brièveté (moins d'une minute et dix secondes) et 
prennent la forme d'un passage de bras en bras entre les parents et les éducatrices en charge des accueils.

Dans le but de mettre en évidence les récurrences et les différences dans les modalités d'accueil d'Axel au fil de la trajectoire d'extraits disponibles, nous restituons sous la forme de vignettes dessinées à partir de captures d'écran certains traits de l'action aux différentes étapes des situations d'arrivées.

\section{a) Les ouvertures des situations d'interactions}

Dans les deux premiers extraits, l'éducatrice, Alex et le parent se trouvent isolés du reste du groupe d'enfants. Lors de l'extrait 3 , de nombreux autres parents sont présents; l'éducatrice $\mathrm{D}$, en charge de l'accueil, arrive en même temps qu'Alex et son père. Dans l'extrait 4 , plusieurs professionnelles sont présentes et l'éducatrice remplaçante (R) qui accueille Alex se trouve installée à une table, en présence d'autres enfants.

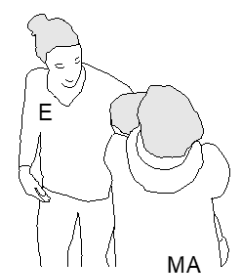

Extrait $1(A)$ $E$ : salut Alex\ça va।

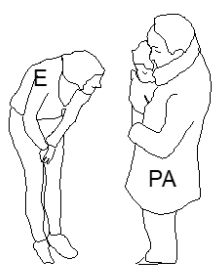

Extrait 2(A)

$\mathrm{E}: \mathrm{SALut}$ Alex comment tu vas:

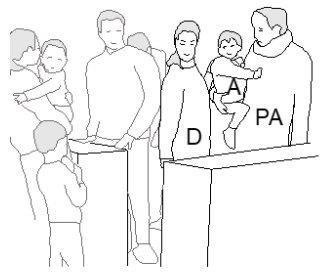

Extrait 3(A)

$D$ : comment ça va/ ((touche le dos de A))

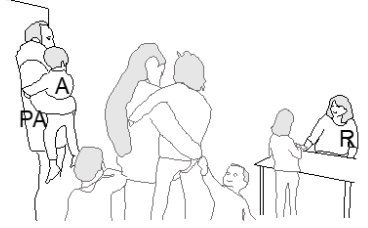

Extrait 4(A)

$\mathrm{R}$ : il va bien. / Alex /

Malgré d'importantes variations contextuelles, de nombreuses similarités se dégagent entre les extraits considérés. Dans chacun des extraits, les participants s'engagent dans un échange confirmatif de salutations, précédé d'un contact visuel réciproque. Pour ce faire, ils commencent par aligner leurs corps pour faciliter un contact visuel entre l'enfant et l'éducatrice (extraits 1, 2, 4). Ils accomplissent ensuite des gestes de toucher au moment d'adresser des salutations à Alex (extraits 1,2,3). Enfin, ces actes verbaux de salutation sont répétés à plusieurs reprises et sont produits différemment selon qu'ils soient adressés aux adultes ou à l'enfant (extraits 1, 2, 3, 4).

\section{b) Les « passages » de l'enfant}

Malgré les contextes très différents, les conduites interactionnelles lors de l'ouverture des séquences créent les conditions d'un alignement des corps et des mouvements des trois participants impliqués permettant à Alex de passer des bras de son parent à ceux des éducatrices :
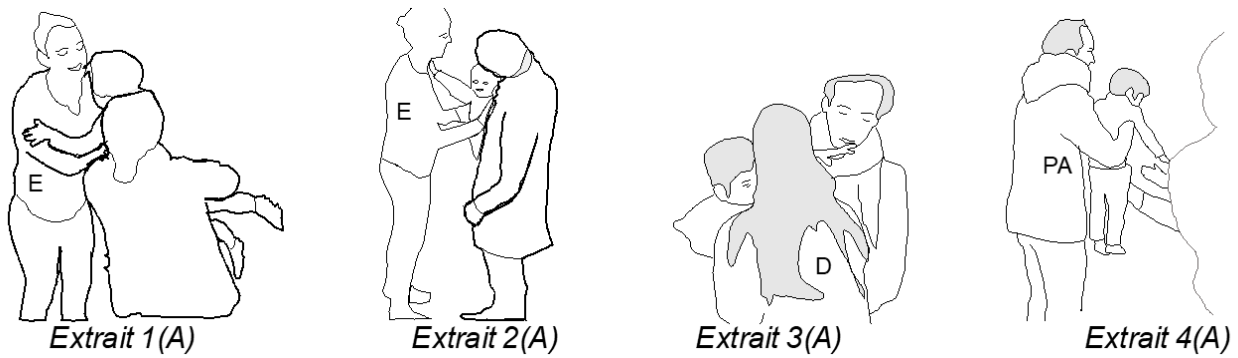

l'éducatrice accomplit le geste d'invitation, auquel Alex s'aligne en tendant ses bras en sa direction. La mère accompagne ce déplacement en soutenant le poids de l'enfant. Lors de l'extrait 2, c'est Alex qui initie le mouvement et qui commence à lever ses mains en direction de l'éducatrice. Dans un enchaînement fluide, l'éducatrice s'aligne à cette conduite de l'enfant et lui présente ses deux mains avec les paumes ouvertes. Lorsque l'éducatrice prend Alex dans ses bras, le père lâche prise immédiatement et permet ainsi à l'éducatrice de saisir l'enfant aisément. L'alignement des trois participants est aussi fluide et rapide que dans l'extrait 1 , mais se réalise avec une distanciation des corps plus importante. Dans l'extrait 3 , le père initie le moment du «passage ». Il embrasse Alex, le soulève en direction de la professionnelle et celle-ci répond à l'invitation par un geste d'accueil, également produit par l'enfant. En soulevant l'enfant, le père d'Alex aménage ici aussi une certaine distanciation des corps entre l'éducatrice et lui. L'initiation du « passage » de l'extrait 4 est également accomplie par le père, qui explicite verbalement à l'éducatrice remplaçante, assise à la table : « désolé pour vou:s .. il veut toujou:rs .. x les bras \». Le père porte alors l'enfant en direction de l'éducatrice, qui tend immédiatement les bras en direction de l'enfant. Lorsque l'éducatrice prend Alex dans ses bras et l'assied sur ses genoux, le père reste à distance avec les bras tendus. leur initiation, la distanciation physique et la présence ou absence de verbalisations. Le passage du premier extrait est initié par l'éducatrice, le deuxième par Alex; le père d'Alex amorce le moment de passage dans les extraits 3 et 4 . La proximité physique est plus importante dans l'extrait 1 , lorsque la mère soutient le poids d'Alex et accompagne son mouvement. Le père en revanche lâche prise immédiatement dans l'extrait 2. Dans les extraits 3 et 4, il tend l'enfant à la professionnelle en charge de l'accueil, ce qui l'amène à rester à une certaine distance physique. Des verbalisations concernant la modalité du passage sont présentes seulement dans l'extrait 4 . Dans les autres cas, le passage est accompli sans faire l'objet d'un commentaire réflexif de la part des participants. interactants permet une coordination fine au moment où Alex est passé de bras en bras. Chacun des participants contribue activement à l'accomplissent de cette action fondamentale du protocole d'accueil. E, l'éducatrice de référence d'Alex, et qui le connait bien, donne le rythme dans les extraits 1 et 2 . Lorsque les professionnelles connaissent moins l'enfant, c'est le parent qui rythme l'échange dans les extraits 3 et 4 . Alex pour sa part ne reste pas inactif dans ces passages et il y contribue ostensiblement par la production de gestes en direction des éducatrices et par des mouvements de son corps qui facilitent le transfert de bras en bras. Ainsi donc, si les modalités de coordination varient, elles procèdent dans l'ensemble des quatre extraits d'ajustements réciproques de l'enfant, de l'éducatrice et du parent.

L'analyse permet également de repérer un geste récurrent dans les extraits concernant les passages d'Alex. Par ce geste, les professionnelles présentent leurs deux mains à l'enfant, paumes ouvertes et dressées vers le haut. Le geste est accompagné par un regard ostensiblement orienté vers l'enfant. 
Figure 4 : Geste d'invitation au passage.

Fig. 4 : gesture inviting the child to pass through

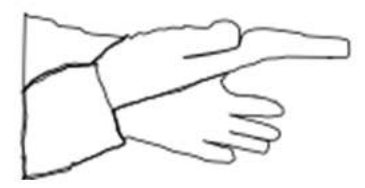

\begin{abstract}
l'enchaînement de mouvements permettant de passer Alex de bras en bras. Il constitue pour ainsi dire l'acte inaugural du moment de passage. Ce geste d'invitation peut être initié par l'éducatrice (extrait 1), consécutif à un geste de l'enfant (extrait 2), ou suivre le mouvement initié par le père, lorsqu'il soulève Alex (extrait 3). Dans le dernier extrait (extrait 4), l'éducatrice est assise et le positionnement des corps modifie les conditions d'effectuation du geste, qui reste cependant présent. Dans les arrivées d'Alex, ce geste peut être placé en première ou en seconde position d'une organisation séquentielle; il peut donc présenter une valeur aussi bien initiative que réactive. Réalisé par des éducatrices différentes, il contribue à accomplir des moments de passage de manière à la fois rapide et fluide, impliquant l'alignement de l'ensemble des participants.
\end{abstract}

\title{
c) Les clôtures de l'interaction
}

Dans les quatre extraits, le moment du passage d'Alex de bras en bras est suivi immédiatement par des salutations de clôture :

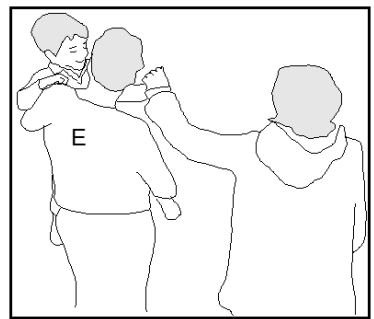

Extrait 1(A)

$E$ : au revoir maman à ce soir

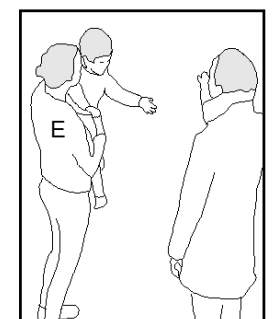

Extrait 2(A)

E : tu dis au revoir à papa/

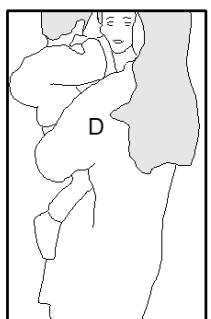

Extrait 3(A)

$\mathrm{D}$ : le dices adios a tu papà

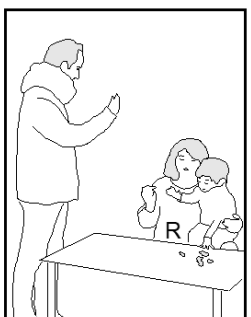

Extrait 4(A)

$\mathrm{R}$ : tu dis au revoir à papa

Dans l'ensemble des situations considérées et indépendamment des participants présents, l'éducatrice incite Alex à dire au revoir à son parent (extraits 1, 2, 3, 4). En réponse à ces invitations, l'enfant et son parent accomplissent des gestes d'au revoir dans les quatre séquences. Dans les extraits 3 et 4 , Alex oriente son attention très rapidement vers les jeux ou les personnes présentes dans l'espace de la structure d'accueil. Dans l'extrait 3, les salutations de clôture sont très brèves. Dans l'extrait 4, l'éducatrice remplaçante fait également des gestes d'au revoir, adressés au père d'Alex, et incite ainsi Alex à l'imiter.

L'analyse de la trajectoire des arrivées d'Alex au fil du corpus disponible permet de souligner le caractère ordonné et récurrent des sortes d'action qui structurent la rencontre entre les participants. Ces rencontres débutent par des échanges d'ouverture et de salutations, elles se poursuivent par un passage rapide de l'enfant de bras en bras et se terminent par des salutations d'au revoir, produits à la fois verbalement et gestuellement. Les récurrences dans la trajectoire des arrivées d'Alex portent ainsi sur 
le positionnement de l'enfant, sur la durée de l'accueil, le rythme de son accomplissement, la succession de phases dans le déroulement de l'interaction et enfin sur l'alignement des mouvements et des corps des trois interactants lors du moment de passage. On peut se demander s'il s'agit ici d'une routine propre à un enfant particulier ou si elle est repérable également auprès d'autres enfants et leurs familles. Comment les routines identifiées dans la trajectoire des accueils d'Alex sont-elles accomplies en présence de participants différents? Prennent-elles une forme similaire ou impliquentelles une adaptation de la part des professionnels?

\subsection{L'analyse d'un cas contrasté : l'accueil Tessa}

Pour faciliter la comparaison, l'analyse porte ici sur un cas singulier, celui de l'accueil de Tessa, qui présente sur le plan contextuel plusieurs similitudes avec les arrivées d'Alex. Cette arrivée de Tessa constitue le troisième extrait en référence à la trajectoire qui sera examinée ultérieurement (voir 4.5). Le choix de la séquence retenue s'effectue par rapport au positionnement de l'enfant, qui ici aussi passe de bras en bras. L'extrait sélectionné implique l'éducatrice $\mathrm{E}$, déjà présente dans deux extraits analysés concernant Alex (extraits 1(A) et 2(A)). Par ailleurs, l'extrait présente une durée comparable (1:07 minutes) avec celle examiné antérieurement à propos d'Alex (voir 4.2). Le geste d'ouverture du moment de passage (voir Figure 4) est également présent. Dans ce contexte, nous proposons d'observer comment cet accueil de Tessa se déroule séquentiellement et en quoi il se différencie ou non de ceux concernant Alex.

L'arrivée de Tessa se passe très tôt le matin. Tessa (T) et sa mère (MT) arrivent lorsque les professionnelles sont encore occupées à la mise en place et à l'aménagement des locaux. Clara (C), l'éducatrice de référence de Tessa est au téléphone et demande à sa collègue Elsa $(\mathrm{E})$ d'accueillir Tessa et sa mère :

Extrait 3(T) : Ouverture de l'interaction

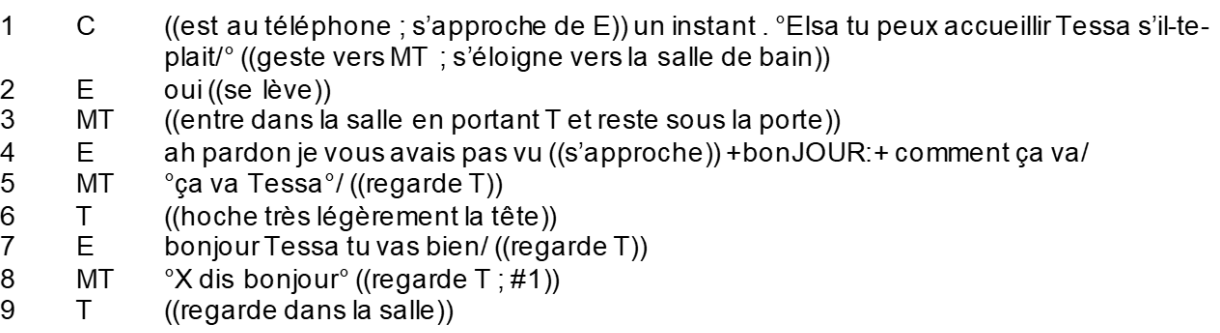

Dans l'extrait retranscrit ci-dessus, l'éducatrice initie un échange de salutation en s'approchant de Tessa et de sa mère (1. 4). En réaction, la mère s'oriente vers son enfant et l'invite à répondre à la question posée en la reformulant (" ça va Tessa/ », 1. 5). Mais Tessa ne répond pas directement à la professionnelle et produit un léger hochement de tête en réaction (1.6). L'éducatrice s'adresse alors spécifiquement à Tessa en la 
désignant comme destinataire ratifiée de ses salutations : «bonjour Tessa tu vas bien/ 》 (1. 7). Ici aussi, la mère reformule la question et encourage Tessa de répondre : « dis bonjour » (1. 8). Lors de ces salutations, il n'y a pas d'échange de regards qui s'établit entre la mère et l'éducatrice ou entre Tessa et l'éducatrice. Tessa et sa mère restent orientées principalement l'une vers l'autre, avec quelques regards d'observation de Tessa vers la salle et vers l'éducatrice qui est au téléphone (\#1). Les échanges de salutations n'aboutissent pas à un alignement simultané entre les trois participants.

\section{Extrait 3(T) : Le « passage » de Tessa}

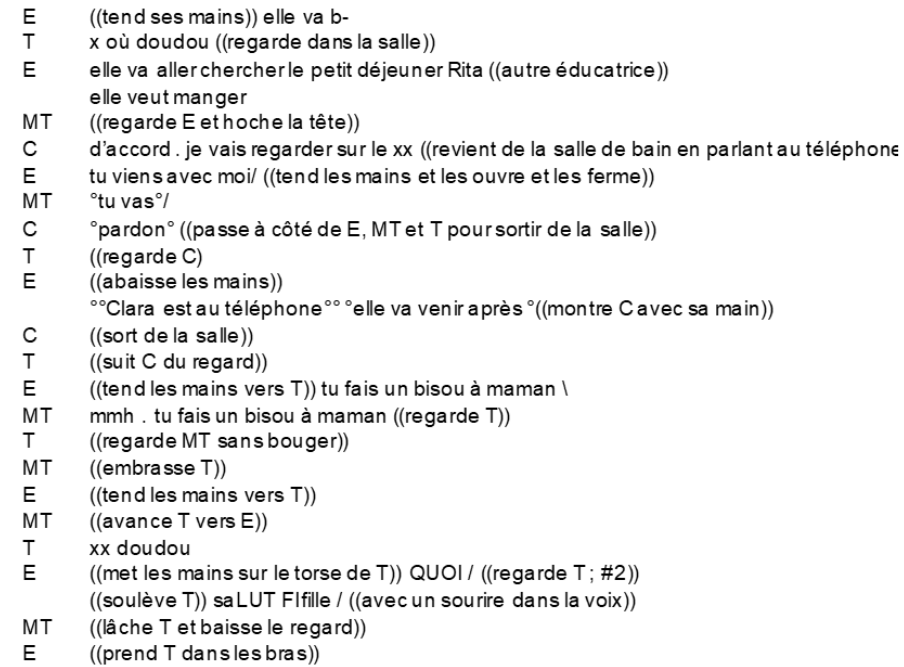

51 Après ces salutations, l'éducatrice présente ses mains à Tessa dans un geste d'invitation à quatre reprises $(1.10,16,24,28)$ et cherche ainsi à initier le moment de passage de Tessa dans ses bras. Tessa et sa mère se n'alignent pas aux trois premières invitations. La première fois, Tessa s'oriente vers la salle (l. 11), la deuxième fois, l'éducatrice qui est au téléphone passe à côté de Tessa et attire son attention (1.19) et la troisième fois Tessa et sa mère s'orientent à se faire un bisou (1. 27). L'éducatrice tend alors ses mains pour la quatrième fois. L'enfant la regarde, mais n'esquisse pas de mouvement. L'éducatrice regarde Tessa, pose ses mains sur le torse de l'enfant, s'arrête pendant un court moment et observe Tessa. Elle soulève alors l'enfant et la prend dans les bras (\#2). Tessa ne résiste pas, mais elle ne tend pas ses bras et ne fait pas de mouvement pour faciliter le passage des bras de sa mère dans les bras de l'éducatrice. Le dessin de la capture d'écran montre également clairement que la mère continue à tenir les jambes de Tessa pendant que l'éducatrice soulève Tessa. Le mouvement de passage de Tessa dans les bras est effectué par l'éducatrice, sans le soutien actif de Tessa ni de sa mère, qui s'y alignent avec un léger décalage temporel. 

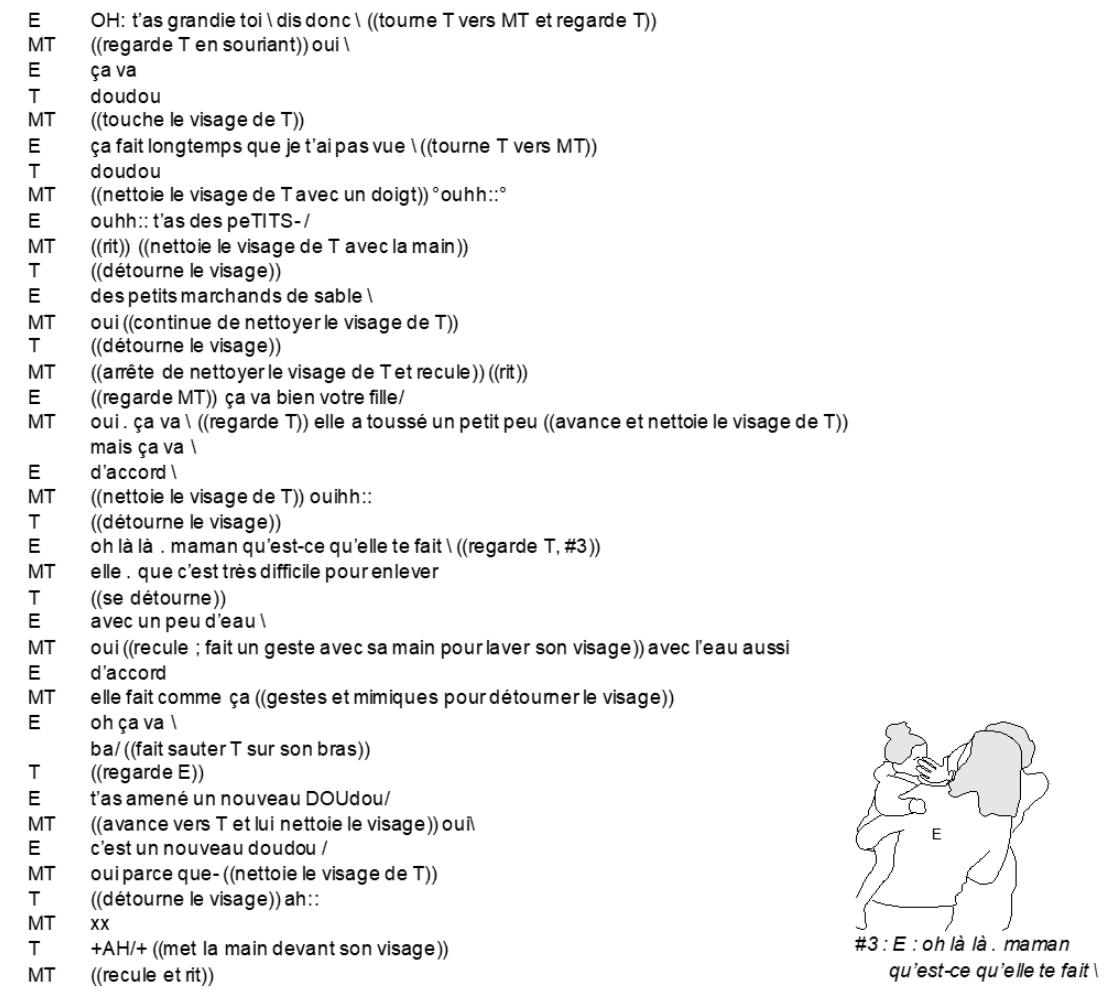

52 Après avoir pris Tessa dans les bras, l'éducatrice ne s'oriente pas immédiatement vers les salutations de clôture. Elle se tourne pour présenter le visage de Tessa face à sa mère, tout en s'adressant à l'enfant : « $\mathrm{OH}$ : t'as grandi toi \dis donc \» (1.35). Par cet énoncé, l'éducatrice rend manifeste qu'elle ne s'occupe pas de Tessa habituellement en exprimant sa surprise concernant un changement intervenu (Tessa a grandi). Cependant, E montre simultanément qu'elle connait Tessa et qu'elle arrive à identifier ce changement. La mère commence alors à nettoyer les yeux et le visage de Tessa.

53 Tessa résiste au nettoyage de son visage, par de petits bruits de protestation et en détournant son visage. Les trois participants s'engagent alors dans des activités multiples et simultanées. La mère de Tessa nettoie le visage de sa fille à quatre reprises $(1.42,47,53,66)$, tout en discutant avec l'éducatrice de la difficulté de laver le visage de Tessa et de la santé de sa fille. Tessa cherche à résister au nettoyage de son visage. L'éducatrice commente les gestes de la mère, rend manifeste qu'elle observe la résistance de Tessa et cherche, à plusieurs reprises, à introduire une nouvelle thématique portant sur l'état de santé de Tessa ou son doudou. Lorsque sa mère recommence à nettoyer son visage pour la quatrième fois, Tessa se détourne vivement et crie de façon très aiguë (1.72). À ce moment-là, l'éducatrice s'oriente vers les salutations et la clôture.

La parenthèse entre le passage de Tessa dans les bras de l'éducatrice et l'initiation de la clôture de l'interaction est d'une durée de 30 secondes. En posant sa main sur le visage de Tessa pendant ce moment, sa mère empêche l'enfant de maintenir un contact visuel avec l'éducatrice ou avec elle-même. Les désalignements entre les participants sont nombreux et concernent les différentes thématiques abordées, mais également les enchaînements séquentiels des tours et des modalités d'adressage. 
Suite au cri de Tessa, l'éducatrice fait sautiller l'enfant dans ses bras en la soulevant par un mouvement bref. La mère recule en direction de la porte :

Extrait 3(T) : La clôture de l'interaction
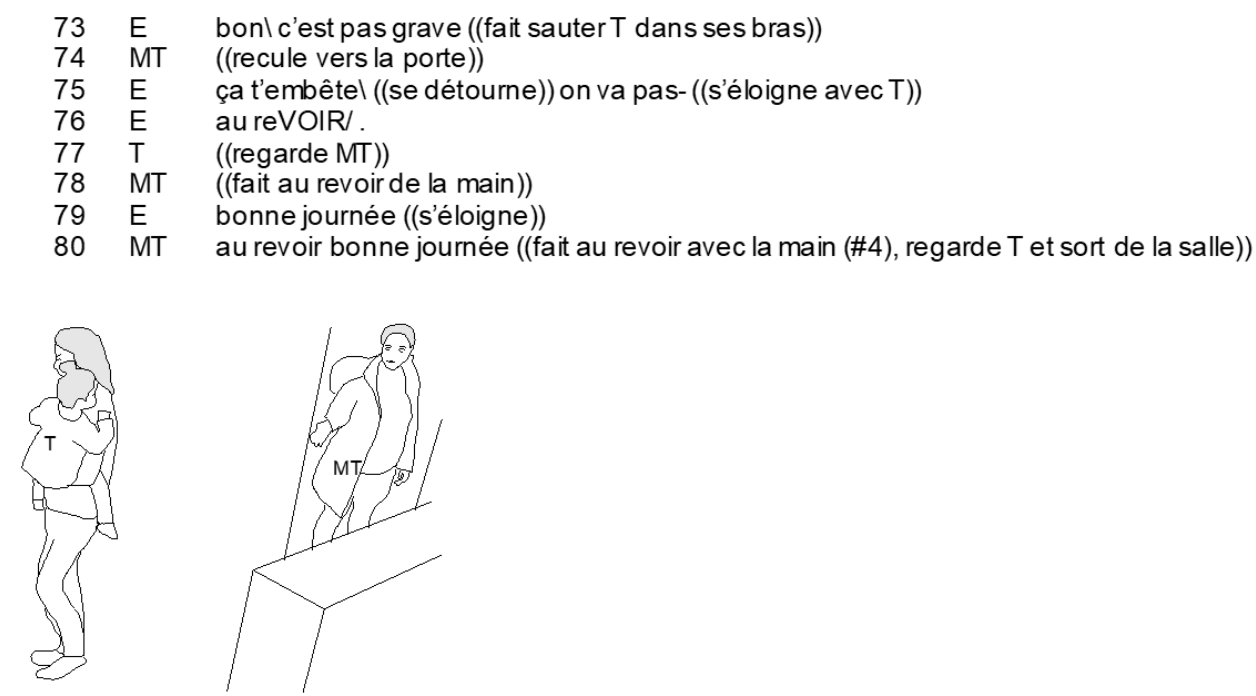

\#4: MT fait au revoir avec la main

La clôture de l'interaction est très rapide dans cet extrait. L'éducatrice s'éloigne avec Tessa en lui parlant (" ça t'embête, l. 75), et en laissant un énoncé en suspens (« on va pas- », 1. 75), avant de saluer la mère. En contraste avec les arrivées d'Alex, l'éducatrice n'incite pas du tout l'enfant à dire au revoir à son parent. Tessa regarde sa mère audessus de l'épaule de l'éducatrice. La mère lui fait un geste d'au revoir de la main. L'éducatrice, qui s'est éloignée, ajoute une dernière salutation, à laquelle la mère répond, avant de sortir de la salle.

La comparaison entre les arrivées d'Alex et celle de Tessa montre des récurrences en ce qui concerne la durée des accueils et le positionnement de l'enfant, qui est passé de bras en bras. De façon analogue, des échanges confirmatifs d'ouverture et de salutations structurent la rencontre entre les participants. En revanche, des différences importantes sont à relever concernant l'alignement ou non des participants et les étapes constitutives du cours de l'interaction. Lors de l'arrivée de Tessa, le moment du passage n'est pas suivi immédiatement par la clôture de l'interaction avec le parent, mais une activité supplémentaire est intercalée, durant laquelle la mère de Tessa continue à nettoyer le visage de sa fille à plusieurs reprises, avant de s'orienter vers son départ. De telles différences se présentent-elles dans toutes les arrivées de Tessa ? Pour répondre à cette question, il nous faut examiner la trajectoire de l'ensemble des situations d'accueil concernant cette même enfant.

\subsection{L'analyse d'une trajectoire contrastée : les accueils de Tessa}

Dans l'ensemble des données filmiques, il est possible d'identifier sept situations d'arrivées exploitables concernant Tessa. Dans ces situations, six éducatrices différentes (E1-E6) accueillent Tessa. Le positionnement de Tessa fluctue dans ces différentes situations. Parfois, elle arrive en marchant et entre dans la structure d'accueil en marchant (extraits 4 et 5); parfois elle arrive en étant portée et elle est passée de bras en bras (extraits 1 et 3). Enfin, il arrive qu'elle soit portée à son arrivée, 
mais qu'elle entre dans la salle en marchant (extrait 6). Dans deux situations d'arrivées, son positionnement fluctue : Tessa est portée par sa mère, posée par terre, reprise dans les bras de sa mère. Dans l'extrait 2, elle entre finalement en marchant dans la salle, tandis que dans l'extrait 7, elle finit d'être passée de bras en bras en pleurant. Les variations des positionnements de Tessa s'observent clairement dans les dessins effectués à partir des captures d'écran :

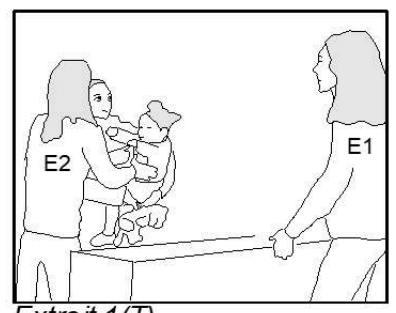

Extrait 1(T)

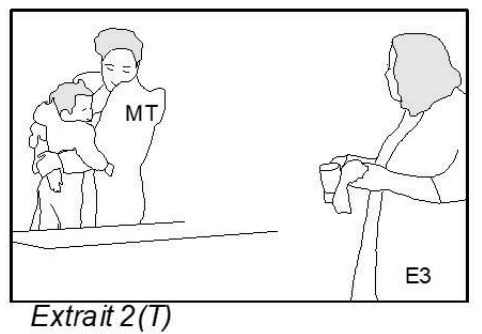

Extrait 2(T)

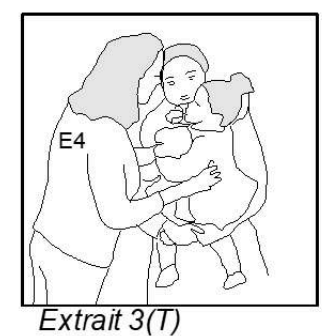

E5

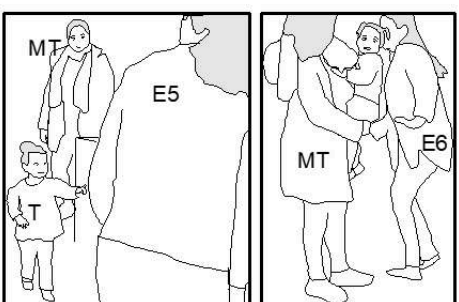

Extrait 7(T)

Une analyse des sept extraits concernant Tessa fait ressortir les nombreuses variations de ces situations, notamment en ce qui concerne les positionnements de Tessa dans l'espace. Les différents positionnements de Tessa peuvent être résumés dans le tableau ci-après :

Tableau 4 : Les variations des positionnements de Tessa. Table 4: Variations in Tessa's positioning

\begin{tabular}{|l|l|l|l|l|l|l|l|l|}
\hline Séquences & 1 & 2 & 3 & 4 & 5 & 6 & 7 \\
\hline Positionnements & M/M (marche \& marche) & & & & & & & \\
\hline & P/P (porté \& porté) & & & & & & & \\
\hline & P/M (porté \& marche) & & & & & & & \\
\hline & Fluctuation s de positionnements & & & & & & & \\
\hline
\end{tabular}

Ces variations se reflètent également dans la durée des séquences. En comparant les arrivées d'Alex et de Tessa, leurs durées varient entre 0:26 et 1:10 minutes pour Alex et entre 1:00 et 3:06 minutes pour Tessa : 
Figure 5 : Comparaison des durées des séquences d'arrivées d'Alex et de Tessa. Figure 5: Comparison of the durations of Alex and Tessa's arrival sequences
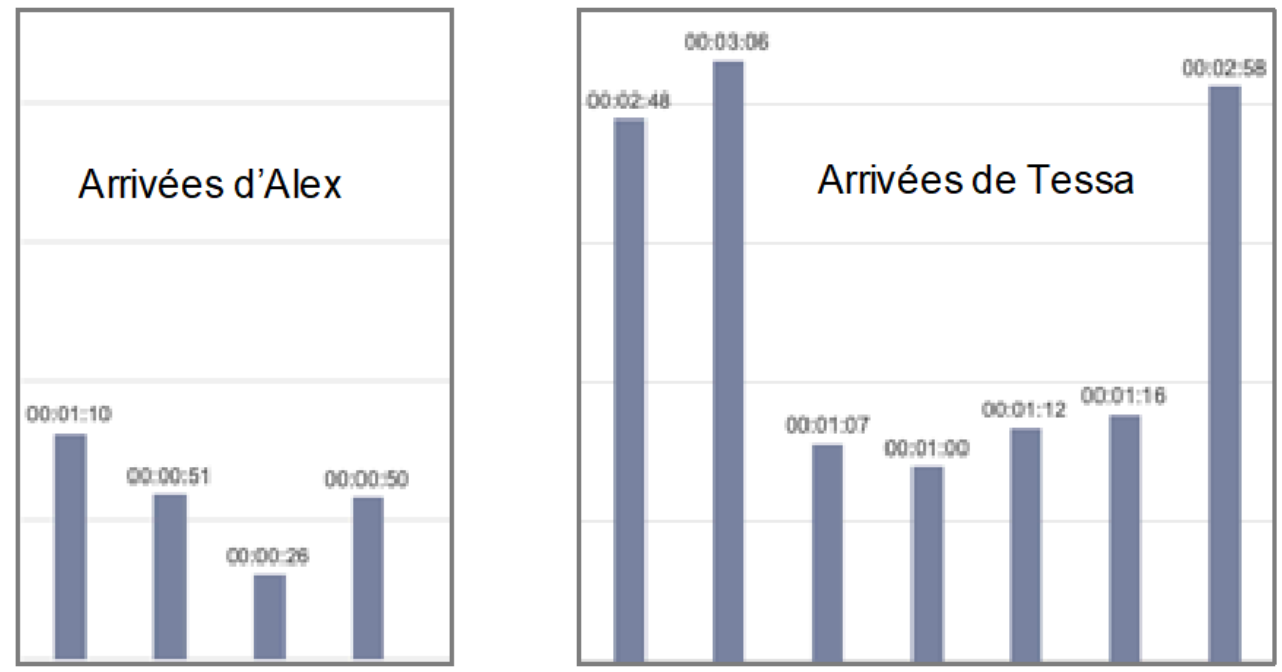

Les trajectoires des arrivées d'Alex et de Tessa se différencient dès lors en ce qui concerne les récurrences ou les fluctuations des positionnements et la variabilité de leurs durées. Les différences portent également sur les alignements des trois participants lors de ces rencontres.

Tableau 5 : Alignement des participants lors des moments de passage. Table 5: Participant alignment when children passing through

\begin{tabular}{|c|c|c|c|c|c|c|c|c|c|c|c|c|c|}
\hline \multicolumn{3}{|c|}{ Alignements } & \multicolumn{4}{|c|}{ Extraits d'Alex } & \multicolumn{7}{|c|}{ Extraits de Tessa } \\
\hline & $1(\mathrm{~A})$ & $2(A)$ & $3(A)$ & $4(A)$ & $1(T)$ & $2(T)$ & $3(T)$ & $4(T)$ & $5(\mathrm{~T})$ & $6(T)$ & $7(T)$ \\
\hline \multirow[t]{4}{*}{$\begin{array}{l}\text { Alignement } \\
\text { simultanée }\end{array}$} & $\begin{array}{l}\text { De } 3 \\
\text { part. }\end{array}$ & $\begin{array}{l}\text { Educatrice, } \\
\text { parent et enfant }\end{array}$ & & & & & & & & & & & \\
\hline & $\begin{array}{l}\text { De } 2 \\
\text { part. }\end{array}$ & $\begin{array}{l}\text { Educatrice et } \\
\text { parent }\end{array}$ & & & & & & & & & & & \\
\hline & & $\begin{array}{l}\text { Educatrice et } \\
\text { enfant }\end{array}$ & & & & & & & & & & & \\
\hline & & Parent et enfant & & & & & & & & & & & \\
\hline \multirow{3}{*}{\multicolumn{2}{|c|}{$\begin{array}{l}\text { Prise d'initiative par } \\
\text { un participant avec } \\
\text { alignem ent possible } \\
\text { postérieur des deux } \\
\text { autres participants }\end{array}$}} & Educatrice & & & & & & & & & & & \\
\hline & & Parent & & & & & & & & & & & \\
\hline & & Enfant & & & & & & & & & & & \\
\hline
\end{tabular}

Lors des arrivées d'Alex, les trois participants s'alignent simultanément sur un même mouvement pour faciliter le passage de l'enfant, avec une coordination fine permettant la participation de chacun. Quant aux arrivées de Tessa, des variations s'observent dans chacun des extraits. Dans certaines situations, deux des participants s'alignent simultanément sur une même conduite, tandis que le troisième participant s'ajuste ultérieurement. Ainsi, dans l'extrait $1(\mathrm{~T})$, la mère discute avec une éducatrice pendant qu'une stagiaire prend Tessa dans ses bras, sans que la mère s'oriente vers son enfant. Dans l'extrait 2(T), une éducatrice propose à boire à Tessa qui cherche alors à descendre des bras de sa mère, qui ne s'y aligne pas immédiatement. Dans l'extrait $7(\mathrm{~T})$, la mère et l'éducatrice se coordonnent pour passer Tessa de bras en bras, malgré la résistance et les pleurs de l'enfant.

Dans d'autres extraits, un participant initie une conduite et les deux autres s'ajustent de façon consécutive. Ceci se constate dans l'extrait 3(T) analysé en détail ci-dessus 
(point 4.4), lorsque l'éducatrice initie le moment de passage. Cependant, c'est souvent Tessa qui initie une conduite, lorsqu'elle va jouer avec un autre enfant par exemple (extraits $4(\mathrm{~T}), 5(\mathrm{~T})$ ), et sa mère et l'éducatrice qui s'y ajustent dans un après-coup. Dans aucun des extraits concernant Tessa, les trois participants s'alignent simultanément lors d'un moment de passage.

Le geste récurrent identifié lors des arrivées d'Alex (voir point 4.3., Figure 4) s'observe également dans certaines des rencontres concernant Tessa (extraits 1(T), 2(T), 3(T), $7(\mathrm{~T})$, mais il n'est pas suivi par un alignement des trois participants. Malgré la présence récurrente d'éléments routiniers lors des arrivées de Tessa, comme les formes ritualisées de salutations lors des ouvertures et des clôtures des situations d'interaction, ces situations d'interactions se caractérisent par une grande variation et une certaine imprévisibilité.

\section{Discussion et conclusion}

Dans cet article, nous avons cherché à mettre en évidence les phénomènes de récurrence et de variation qui sont susceptibles de survenir lorsque sont accomplis des processus d'interaction présentant un haut degré de routinisation. Dans le cas des arrivées des enfants au sein des structures d'accueil, deux trajectoires d'accueil ont été identifiées et décrites avec la même systématique, permettant de dégager des relations à la fois d'invariance et de variation. Les situations d'accueil d'Alex présentent un haut degré de fluidité et de récurrence. Les conditions dans lesquelles elles sont accomplies n'exercent que peu d'influence sur les moments repérables qui constituent les rencontres sociales et sur la manière dont sont mises en œuvre les ressources permettant de les accomplir, et ce indépendamment des adultes concernés. Dans l'ensemble des extraits considérés, l'engagement des participants se caractérise par un fort alignement des participants et un haut degré de coordination entre eux. La trajectoire des arrivées de Tessa présente moins de stabilité et de prévisibilité. Les sortes d'action qui s'y trouvent collectivement accomplies varient, tout comme les conditions spatiales d'arrivée et de passage de l'enfant. Le degré d'acceptation de l'enfant quant au moment opportun pour "passer » dans l'espace de l'institution est fluctuant, tout comme la capacité des participants à s'ajuster à leurs conduites réciproques. Dans ces conditions, les déroulements de ces arrivées sont appelés à varier dans leur durée et dans les méthodes au moyen desquelles ils sont accomplis en situation.

Ainsi donc, certaines conduites interactionnelles se caractérisent par des récurrences et peuvent être désignées comme des «routines» interactionnelles. Les traits emblématiques de telles routines portent sur l'agencement des ouvertures, des moments de passages et des clôtures des interactions avec les parents et les enfants lors de leur arrivée. Les ouvertures s'accomplissent par la répétition des salutations (répétitions des «bonjours » et des « ça va »), par l'alignement des regards et des corps ainsi que par le toucher. Les moments de passage routinisés se caractérisent par une coordination fine et l'alignement simultané des trois participants sur un même mouvement. La prise d'initiative peut être distribuée sur un ou plusieurs des participants. Il existe des gestes emblématiques qui semblent constituer des indicateurs pour l'ouverture $\mathrm{du}$ moment $\mathrm{du}$ passage. Ces moments montrent une forte interdépendance entre les trois participants. Les temps de clôture s'accomplissent par 
des gestes récurrents (geste d'" au revoir »), des salutations avec des adressages directs et indirects, et des échanges de regards nombreux. Les répétitions des salutations permettent d'observer à plusieurs étapes du processus de clôture les alignements de l'enfant et, ainsi, de prendre en compte l'« état » de l'enfant et l'orientation de son attention.

Pourtant, comme les analyses le montrent, un alignement simultané des trois participants n'est pas observable dans toutes les arrivées. Différentes modalités de synchronisation ou de dé-synchronisation peuvent de présenter. Lorsque les alignements ne s'accomplissent pas de façon simultanée, des éléments routinisés restent malgré tout présents et sont mobilisés pour s'engager dans des processus de réparation ou de négociations. Pour permettre une coordination malgré les désalignements, les participants effectuent par exemple des répétitions (répétitions de "gestes d'invitations, de salutations, de demandes, etc.). Les verbalisations permettent parfois d'annoncer des conduites ultérieures ou d'expliciter des ajustements. L'échange de regards est à la base de processus d'observation qui se décèlent également lors des silences ou des ralentissements dans le flux interactionnel. L'aménagement d'activités secondaires permet de constituer des "parenthèses » lorsqu'une conduite initiée n'est pas reprise par les autres participants. Par ailleurs, l'accomplissement des arrivées se réalise par le maintien de la progressivité de l'interaction, lorsqu'un des participants initie une conduite et passe d'une étape à une autre. Ces réparations ou négociations sont fréquemment accomplies par l'éducatrice, mais elles peuvent également être prises en charge par les parents.

68 Les mécanismes de synchronisation et de désynchronisation observables dans les rencontres sociales récurrentes illustrent de manière emblématique la double nature, à la fois schématique et émergente de l'ordre de l'interaction. Parce qu'elles reposent sur des processus de typification et qu'elles sont reconnaissables comme une expérience cadrée d'un certain type, les situations d'arrivées des enfants dans les structures d'accueil sont susceptibles de produire des routines qui en facilitent les conditions d'accomplissement. Mais parce que ces interactions émergent dans des circonstances toujours singulières, elles exigent des participants qu'ils s'ajustent en permanence aux contingences qu'ils rencontrent en contexte.

Pour mettre en évidence ces logiques de récurrence et de singularité, des dispositifs méthodologiques spécifiques méritent d'être explorés à partir d'une perspective interactionnelle en analyse du travail. Dans la démarche présentée ici, une combinaison d'observations quantitatives et qualitatives a été proposée. La démarche a montré aussi que pour cerner pleinement la dimension « routinisée » de l'activité, des analyses de cas singuliers sont à la fois nécessaires et non suffisantes. Il importe en effet d'inscrire l'analyse interactionnelle multimodale dans une triple ouverture. Celle d'abord de la création de "collections", permettant de constituer et d'organiser des données empiriques relatives à des familles de phénomènes présentant des caractéristiques proches. Celle ensuite d'une analyse de «trajectoires» de situations, permettant de cerner les conditions d'organisation d'une même activité dans son déploiement temporel (de Saint-Georges \& Filliettaz, 2008). Et celle enfin d'une analyse contrastive, dont l'objectif est de mettre en évidence des phénomènes de ressemblance et de variation entre les extraits d'une collection ou des trajectoires d'extraits. C'est au carrefour entre ces différentes logiques d'analyse qu'est susceptible d'émerger la 
véritable valeur d'une routine interactionnelle, à savoir sa réplicabilité d'une part, et son adaptabilité d'autre part.

Les développements méthodologiques proposés dans cet article sont susceptibles de porter un regard approfondi et renouvelé sur le travail des professionnels de l'éducation de l'enfance, en particulier dans son interface avec la sphère familiale et les échanges avec les parents. La construction ou la re-construction continuelle d'un caractère routinisé des situations d'arrivées amène à un alignement des conduites, accomplies de façon simultanée ou décalée dans le temps. Aussi le travail relationnel avec les parents s'inscrit-il irrémédiablement dans une forme d'intelligence temporelle: il s'agit de trouver le «bon moment" pour accueillir l'enfant, dans un contexte qui se répète quotidiennement, mais qui est susceptible de varier de manière continue. Dans des contextes où l'action sur la progression de la temporalité est distribuée sur plusieurs participants, les interactants sont amenés à synchroniser une pluralité de processus temporellement ordonnés, impliquant le temps de chacun des participants, le temps de l'action et le temps de l'institution. Ici, la compétence des éducatrices et des éducateurs ne consiste donc pas à savoir accomplir des routines interactionnelles préfabriquées ni même à suivre aveuglément un canevas ou un script. Il s'agit plutôt de se servir des routines interactionnelles comme de ressources, auxquelles les partenaires de l'interaction peuvent s'aligner ou pas, selon leurs particularités propres ou leur sensibilité aux circonstances locales. C'est là un exemple emblématique de la mobilisation d'une compétence interactionnelle, essentiellement distribuée sur l'ensemble des participants impliqués, et qui ne peut être développée que dans une logique intersubjective.

Une compréhension fine de cette dimension collective des interactions offre des opportunités de formation. Lors des dispositifs de formation réalisés, les éducatrices ont analysé plusieurs séquences d'arrivées et de départs et elles se sont notamment intéressées à l'interdépendance des conduites des parents, des enfants et des éducatrices et éducateurs (Garcia \& Filliettaz, 2020). Par ailleurs, elles abordent les liens entre les pratiques professionnelles routinisées et les ajustements observables dans les extraits de films. Leur analyse porte majoritairement sur les particularités de situations singulières. Mais, à certains moments, les éducatrices observent aussi une pratique professionnelle précise pour relever qu' « on le fait souvent » ou évoquer ce qui se fait « en général » (Filliettaz \& Zogmal, à paraître). Les analyses détaillées contribuent ainsi à débattre de l'accomplissement du travail et des finalités d'un métier. Des séances de restitution avec l'ensemble des équipes éducatives concernées permettent ensuite aux participantes de présenter leurs analyses et de partager leurs réflexions avec leur collectif de travail. Les démarches de formation déployées montrent que l'analyse des interactions peut constituer une ressource théorique et méthodologique pour identifier et développer la compréhension du déroulement des interactions, et notamment de leurs composantes routinières et émergentes. Un tel regard analytique sur le travail auprès des parents et son accomplissement dans les interactions peut ainsi contribuer à enrichir la formation initiale et continue des éducatrices et éducateurs de l'enfance. 


\section{BIBLIOGRAPHIE}

Alonso Vilches, V., \& Pirard, F. (2016). L'entretien de tutorat documenté : un accompagnement réflexif ? Éducation permanente, 206, 131-138.

Bange P. (1992). Analyse conversationnelle et théorie de l'action. Paris : Hatier-Didier.

Bonnabesse, M., \& Blanc, M. (2013). Penser les relations entre parents et professionnels comme un élément essentiel de la qualité. In S. Rayna \& C. Bouve (Eds.), Petite enfance et participation : Une approche démocratique de l'accueil (pp. 105-124). Toulouse : ERES.

Bouve, C. (2009). Un enjeu de la coéducation : pour une éthique de la rencontre. In S. Rayna, C. Bouve, \& P. Mosset (Eds.), Pour un accueil de qualité de la petite enfance : quel curriculum ? (pp. 281-292). Toulouse : ERES.

Bucholtz, M., \& Hall, K. (2005). Identity and interaction: a sociocultural linguistic approach. Discourse Studies, 7(-5), 585-614.

Brougère, G. (Ed.). (2010). Parents, pratiques et savoirs au préscolaire. Bruxelles : Peter Lang.

Brougère, G. (2015). Learning by participating: A theoretical configuration applied to French cooperative day care centres. In L. Filliettaz \& S. Billett (Eds.), Francophone perspectives of learning through work (pp. 71-93). Dordrecht : Springer.

Chatelain-Gobron, S. (2014). Les retransmissions journalières : de la banalité du quotidien à la complexité de la rencontre. In G. Meyer \& A. Spack (Eds.), Accueil de la petite enfance : comprendre pour agir (pp. 143-164). Toulouse : ERES.

De Saint-Georges, I., \& Filliettaz, L. (2008). Situated trajectories of learning in vocational training interactions. European Journal of Psychology of Education, XXIII(2), 213-233.

Filliettaz, L. (2014). L'interaction langagière : un objet et une méthode d'analyse en formation d'adultes. In J. Friedrich \& J. Pita, J. (Eds.), Recherches en formation des adultes : un dialogue entre concepts et réalité (pp. 127-162). Dijon : Éditions Raison et Passions.

Filliettaz, L. (2018). Interactions verbales et recherche en éducation : principes, méthodes et outils d'analyse. Université de Genève : Carnets des sciences de l'éducation.

Filliettaz, L., \& Zogmal, M. (éd.) (2020). Mobiliser et développer des compétences interactionnelles en situation de travail éducatif. Toulouse : Éditions Octarès.

Filliettaz, L. \& Zogmal, M. (à paraître). Travailler sous le regard d'autrui ; une compétence interactionnelle. Revue des sciences de l'éducation.

Garcia, S. (2021a). Penser les continuités entre recherche et formation professionnelle dans les métiers socio-éducatifs : le cas des compétences interactionnelles des éducateurs de l'enfance dans la relation avec les parents. Recherche \& Formation, 94.

Garcia, S. (2021b). Interagir au travail et en situation de formation : le cas des relations avec les parents dans le champ de l'éducation de l'enfance, Savoirs, 56.

Garcia, S., \& Filliettaz, L. (2020). Compétences interactionnelles et relations des éducateurs de l'enfance avec les parents : la formation comme ressource pour la recherche. Phronesis, 9(2), 123-138. 
Garcia, S., Wolter, L., \& Filliettaz, L. (à paraître). Travailler « sur le pas de la porte » : Le cas des interactions avec les parents dans le champ de l'éducation de l'enfance. Revue d'Anthropologie de la Connaissance.

Garfinkel, A. (2007). Recherches en ethnométhodologie (trad. par M. Barthélémy, B. Dupret, J.M. de Queiroz et L. Quéré). Paris : Presses universitaires de France.

Goffman E. (1973a). La mise en scène de la vie quotidienne, tome 1 : La présentation de soi. Paris : Éditions de Minuit.

Goffman E. (1973b). La mise en scène de la vie quotidienne, tome 2 : Les relations en public. Paris : Éditions de Minuit.

Goffman E. (1974). Les rites d'interaction. Paris : Éditions de Minuit.

Goffman, E. (1988). La situation négligée (trad. par Y. Winkin). In Y. Winkin (Ed.), Erving Goffman : les moments et leurs hommes (pp. 143-149). Paris : Édition du Seuil et Éditions de Minuit.

Goffman, E. (1991). Les cadres de l'expérience (trad. par I. Joseph avec M. Dartevelle et P. Joseph). Paris : Éditions de Minuit.

Goodwin, C. (2000). Action and embodiment within situated human interaction. Journal of Pragmatics, 32, 1489-1522.

Kress, G., Jewitt, C., Ogborn, J., \& Charalampos, T. (2001). Multimodal teaching and learning. The rhetorics of the science classroom. Londres : Continuum.

Lussi Borer, V., Durand, M., \& Yvon, F. (Eds.). (2015). Analyse du travail et formation dans les métiers de l'éducation. Bruxelles : De Boeck Superieur.

McNeill, D. (Ed.) (2000). Language and Gesture. Cambridge : Cambridge University Press.

Mondada, L. (2006). L'ordre social comme un accomplissement pratique des membres dans le temps. Médias et Culture, 2, 85-119.

Mondada, L. (2008). L'analyse de « collections » de phénomènes multimodaux en linguistique interactionnelle : à propos de l'organisation systématique des ressources gestuelles en début de tour. Cahiers de praxématique, 50, 21-66.

Mondada, L. (2017). Le défi de la multimodalité en interaction. Revue française de linguistique appliquée, XXII(2), 71-87.

Pastré, P. (2011). La didactique professionnelle. Paris : Presses universitaires de France.

Pekarek Doehler, S., Bangerter, A., de Weck, G., \& Fillettaz, L. (Eds.). (2017). Interactional competences in institutional settings: From school to the workplace. Londres : Palgrave Macmillan.

Plan d'études cadres (PEC) (2015). Éducatrice de l'enfance ES, Éducateur de l'enfance ES. SPAS/OrTraS.

Pirard, F. (2007). L'accompagnement professionnel face aux enjeux de qualité de services. In G. Brougère \& M. Vandenbroeck (Eds.), Repenser l'éducation des jeunes enfants (pp. 225-243). Bruxelles : Peter Lang.

Pirard, F., \& Barbier, J.-M. (2012). Accompaniment and quality of childcare services: the emergence of a culture of professionalization. Early Years, 32, 169-182.

Quéré L. (1990). Agir dans l'espace public. L'intentionnalité des actions comme phénomène social. In P. Pharo \& L. Quéré (Eds.), Les formes de l'action (pp. 85-112). Paris : EHESS.

Rayna, S., Rubio, M.-N., \& Scheu, H. (Eds.) (2010). Parents-professionnels : la coéducation en questions (pp. 37-48). Toulouse : ERES. 
Relieu, M., Salembier, P., \& Theureau, J. (Eds.) (2004). Activité et action/cognition située. Revue électronique Activité, 1(2). https://journals.openedition.org/activites/1179.

Sacks, H. (1992). Lectures on conversation [2 vol.]. Oxford : Blackwell.

Sacks, H., Schegloff, E., \& Jefferson, G. (1978). A simplest systematics of the organization of turn taking for conversation. In J. Schenkein (Ed.), Studies in the organization of conversational interaction (pp. 7-55). New York : Academic Press.

Schank, R.C., \& Abelson, R.P. (1977). Scripts, Plans, Goals and Understanding. An Inquiry into Human Knowledge Structures. Hillsdale: Erlbaum Associates.

Schegloff, E. A. (1993). Reflections on quantification in the study of conversation. Research on Language and Social Interaction, 26(1), 99-128.

Schegloff, E. (2007). Sequence organization in interaction: A primer in conversation analysis. Cambridge : Cambridge University Press.

Schütz A. (1987). Le chercheur et le quotidien. Phénoménologie des sciences sociales. Paris : Méridiens Klincksieck.

Stokoe, E. (2014). The Conversation Analytic Role-play Method (CARM): A method for training communication skills as an alternative to simulated role-play. Research on Language and Social Interaction, 47(3), 255-265.

Suchman, L.A. (1987). Plans and Situated Actions. The Problem of Human-Machine Communication, Cambridge : Cambridge University Press.

Ulmann, A. (2013). Le travail en crèche à partir de l'invisible. Nouvelle revue de psychosociologie, 15(1), 193-206.

Ulmann, A. (2017). Le travail auprès des jeunes enfants : quels apprentissages pour quelles pratiques professionnelles? Revue française des affaires sociales, 316-327.

Vinatier, I. (2013). Le travail de l'enseignant : une approche par la didactique professionnelle. Bruxelles : De Boeck.

Von Cranach M., Kalbermatten, U., Indermühle, K., \& Gugler, B. (1982). Goal-directed Action: Londres : Academic Press.

Wolter, L. (2020). L'analyse interactionnelle comme levier de compréhension du caractère complexe et invisible du travail : le cas de l'éducation de la petite enfance. Mémoire de Master, Université de Genève.

Young, R.F., \& Miller, E.R. (2004). Learning as changing participation: Discourse roles in ESL writing conferences. The Modern Language Journal, 88(4), 519-535.

Zogmal, M. (2020a). Savoir voir et faire voir : Les processus d'observation et de catégorisation des enfants comme outil de travail dans les pratiques professionnelles des éducatrices et éducateurs de l'enfance. Berne : Peter Lang.

Zogmal, M. (2020b). Apprendre dans des interactions avec de multiples participants : la formation pratique des éducatrices et éducateurs de l'enfance ». Activités, 17(1), consulté le 04 juillet 2020. URL : http://journals.openedition.org/activites/5029

Zogmal, M., \& Durand, I. (2020). De l'observation des enfants à l'analyse interactionnelle : contributions de la recherche à la formation continue des éducateurs et éducatrices de l'enfance. Phronesis, 9(2), 108-122. 


\section{ANNEXES}

\section{Conventions de transcription}

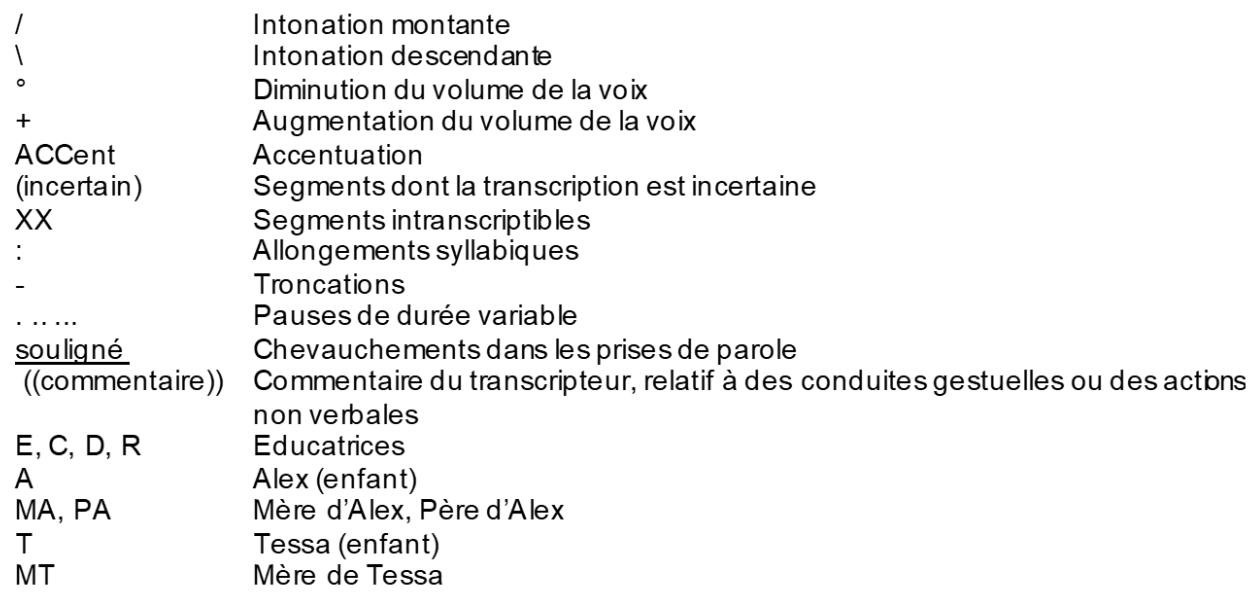

\section{RÉSUMÉS}

Cette contribution s'intéresse aux routines interactionnelles qui structurent le travail des éducatrices et des éducateurs à l'occasion des temps d'accueils des enfants dans leur lieu d'accueil, au moment où ils arrivent dans l'espace éducatif accompagnés de leurs parents. Il vise à mieux comprendre comment se coordonnent les participants au moment de ces accueils et les ressources qu'ils mobilisent dans le cadre de cette coordination. Pour mettre en évidence des éléments récurrents et identifier des routines interactionnelles, la démarche adoptée cherche, plus généralement, à présenter et discuter des voies méthodologiques possibles. En continuité avec des travaux portant sur l'analyse interactionnelle, un dispositif empirique documente de manière méthodique des interactions verbales et non-verbales prenant place au moment des accueils et des départs des enfants et de leurs familles au sein de deux institutions de la petite enfance. Les temps d'accueil sont analysés, dans leur dimension à la fois temporelle et multimodale, au moyen d'une méthodologie qui se déploie par étapes. Des analyses portant sur des séries de situations contrastées et leurs caractéristiques sont complétées par des descriptions fines de cas singuliers. La discussion conclusive permet de revenir sur les ingrédients des routines d'interaction et de discuter leur rôle dans l'organisation du travail des professionnels de l'éducation de l'enfance.

This contribution focuses on the interactional routines that structure the work of educators when children arrive at an early childcare facility, accompanied by their parents. It aims to gain a better understanding of how the participants coordinate themselves during these drop-off situations and the resources they mobilise in the context of these brief encounters. In order to highlight recurrent elements and identify interactional routines, the approach seeks to present and discuss specific methodological procedures. In continuity with research on interactional analysis in professional contexts, empirical data were collected, consisting in video-recorded verbal and non-verbal interactions taking place when children and their families arrive at two early childhood institutions. These drop-off situations are analysed in both their temporal and multimodal dimensions, using a step-by-step methodology. Detailed descriptions of individual cases are combined with analyses of series of contrasting situations and their characteristics. To 
conclude, the ingredients of interactional routines are discussed, as is their role in the organisation of the work of early childhood educators.

\section{INDEX}

Keywords : early childhood education, workplace learning, interaction analysis, interactional routines

Mots-clés : petite enfance, formation d'adultes, analyse des interactions, routines interactionnelles

\section{AUTEURS}

\section{MARIANNE ZOGMAL}

Université de Genève, Interaction \& Formation, Case postale, 1211 Genève Marianne.Zogmal@unige.ch

\section{LAURENT FILLIETTAZ}

Université de Genève, Interaction \& Formation, Case postale, 1211 Genève

Laurent.Filliettaz@unige.ch 